\title{
Versioni e formae vulgatae degli oracoli di Delfi: alcune note a proposito di Erodoto e dei frammenti di Enomao di Gadara
}

Cristian Mondello

\section{Versions and formae vulgatae of the oracles of Delphi: some notes about Herodotus and the fragments of Oenomaus of Gadara}

\begin{abstract}
This paper aims to analyze the relationship between the Histories of Herodotus and the ex-

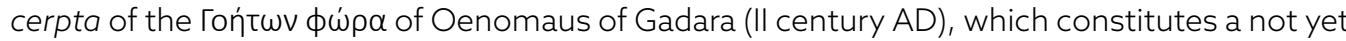
adequately-studied case in the context of the documentation concerning the oracles of Delphi. The goal is to determine the genesis and the developments of these two literary traditions, rejecting those hermeneutic approaches which connected the main source of Oenomaus to Herodotus. The textual comparison of the versions transmitted by the two authors allows to identify a set of lexical and syntactic differences, mutual lacunae and additions of verses, revealing a distinct appearance of the two oracular traditions in terms of chronology, origin and inclinations.
\end{abstract}

\section{Keywords}

oracles; responses; prediction; Delphi; Herodotus; Oenomaus; Diodorus; versions; texts; chresmologues 
Un commentatore antico e irriverente dei responsi profetici dell'oracolo di Delfi fu Enomao di Gadara, filosofo cinico vissuto probabilmente nel II sec. d.C., la cui personalissima opera, dal titolo Гó́ $\tau \omega \nu \varphi \omega \hat{Q} \alpha$, ci è giunta in frammenti grazie al lavoro e all'interesse di un altro celebre dileggiatore dell'oracolistica pagana, quale Eusebio di Cesarea. ${ }^{1}$

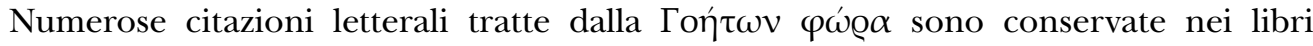
V e VI della Praeparatio Evangelica e riproducono, negli estratti selezionati da Eusebio, parte della polemica antioracolare e di quella antifatalistica che il filosofo cinico aveva condotto nel suo trattato. In particolare, sono riconoscibili due distinti nuclei tematici nel gruppo omogeneo di frammenti conservati nel V libro (capitoli 19-36), i quali costituiscono diciotto estratti nell'edizione Vallette, intervallati da quattordici brevi interventi di Eusebio: nel primo è la serrata polemica di Enomao contro la mantica delfica, svolta attraverso il minuzioso esame di un elevato numero di profezie di Apollo Pitico; nel secondo, la medesima critica è confermata e sostenuta mediante la narrazione dell'esperienza, amara quanto istruttiva, maturata dal filosofo cinico in occasione di una serie ripetuta di responsi ricevuti da Apollo Clario. Il lungo estratto citato da Eusebio nel capitolo 7 del libro VI conserva, invece, la confutazione che il Cinico aveva sostenuto contro il concetto di fato inteso come concatenazione di eventi, secondo la definizione

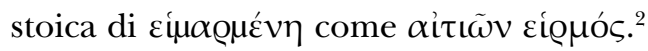

Questi frammenti sono situati da Eusebio in un'ampia digressione della Praeparatio Evangelica in cui si sottolinea, quale effetto della venuta del Salvatore, la fine degli dèi, dei demoni e degli oracoli ingannevoli dei quali, proprio attraverso le formulazioni della

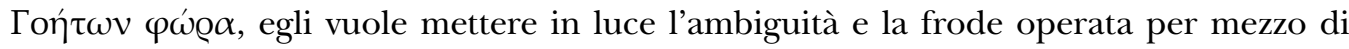
impostori. Il vescovo palestinese presenta lo scritto di Enomao come un'opera senza dubbio personale, contrassegnata da un'argomentazione vigorosa $(\dot{\alpha} v \delta \varrho \iota \kappa \tilde{\omega} \lambda \text { o } \gamma \iota \sigma \mu \tilde{\omega})^{3}$

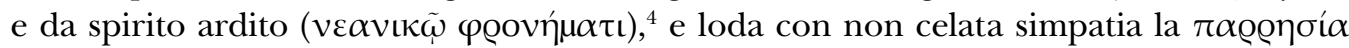

1 Su Enomao di Gadara, contemporaneo forse di Adriano e di Antonino Pio, la bibliografia non risulta essere particolarmente ricca. Tra gli studi più importanti cfr. Saarmann (1887), Dudley (1937: pp. 162-170),

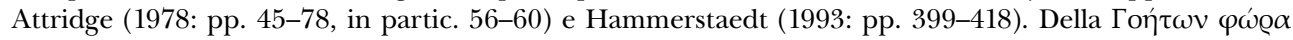
di Enomao di Gadara sono consultabili due raccolte specifiche di frammenti, ovvero quella con traduzione latina, commento e studio introduttivo di Vallette (1908), e quella in lingua tedesca con commento e introduzione a cura di Hammerstaedt (1988). Gli excerpta di Enomao di Gadara sono collezionati anche nella più generale raccolta di frammenti di filosofi greci a cura di Mullach (1867). Sul testo di Enomao si vedano, inoltre, le edizioni della Praeparatio Evangelica: Mras (1954-1956 [1982²]); des Places (1980);

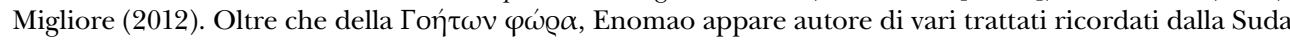

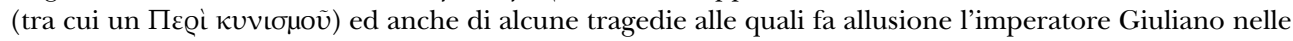
sue Orazioni: cfr. des Places (1980: pp. 7-16).

2 Sull'adesione di Enomao alla linea diogeniana del cinismo e sulle sue personali riformulazioni, specie nel contesto della polemica anti-stoica dei primi secoli dell'età imperiale, cfr. Brancacci (2000: pp. 37-67) e Brancacci (2001: pp. 71-110). Eusebio non esita a citare la critica fatta da Enomao al concetto di

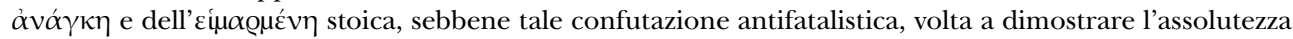
della libertà dell'uomo inteso come causa e produttore di eventi, potesse intaccare la dottrina cristiana della Provvidenza: Vallette (1908: p. 139). A tal proposito si veda des Places (1980: p. 11): “[...] un platonicien comme lui (sc. Eusèbe), qui cite in extenso (P.E. XII, 52, 1-31) la démonstration platonicienne de la Providence, ne craignait pas plus que Platon pour celle-ci les attaques dirigées contre la divination".

3 Eus. p.e. 5, 18, 6, 2.

4 Ivi $6,6,74,2$. 


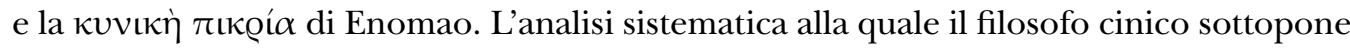
un lungo elenco di responsi (storici e non) dell'oracolo di Delfi introduce al problema dell'uso di Erodoto come una delle fonti presunte di Enomao. Secondo quanto sostenuto da vari interpreti, le Storie erodotee sarebbero da considerarsi come il testo di riferimento impiegato da Enomao in una serie di oracoli delfici riportati nella Гớ $\tau \omega v$ $\varphi \omega ́ \varrho \alpha .{ }^{5}$ In realtà Enomao non cita mai Erodoto nei passi indicati, né chiarisce quali siano le fonti da lui utilizzate nella sua trascrizione o libera rielaborazione dei vaticini delfici. Tale problematica, che non pare sia stata sollevata fino ad oggi nell'ambito della critica, ${ }^{6}$ presenta delle importanti implicazioni non solo riguardo alla scelta ed all'interpretazione degli oracoli citati da Enomao, ma anche relativamente alla genesi e agli sviluppi della tradizione letteraria degli oracoli di Delfi fino all'età tardoantica.

L'obiettivo della presente ricerca è quello di investigare il rapporto tra le versioni degli oracoli delfici riportate da Erodoto e da Enomao di Gadara, nel tentativo di rintracciare le relazioni ma anche le differenze significative rispecchiate dalle tradizioni rispettive dei due autori. ${ }^{7}$ Lo studio è condotto attraverso la comparazione testuale e filologica dei testi tratti dalle Storie di Erodoto e dai frammenti di Enomao conservati da Eusebio ${ }^{8}$ riguardanti gli oracoli delfici comuni, i quali vengono citati secondo la classificazione del catalogo di H. W. Parke e D. E. W. Wormell. ${ }^{9}$ A questo livello di confronto testuale si accompagna l'esame incrociato con gli altri testimonia superstiti, il quale contribuisce alla comprensione di una complessa stratigrafia di fonti, lasciandone emergere rami diversi per cronologia, provenienza e tendenze storico-letterarie. ${ }^{10}$

5 Lo Stellenregister dell'edizione di Mras (1956) indica la voce "Herodotus" come fonte di Enomao di Gadara nei rispettivi passaggi. Anche des Places (1980: p. 11), seguito da Brancacci (2000: p. 40), ritiene Erodoto, accanto a Pausania e ad altri autori, come una delle fonti di Enomao per la serie di oracoli trascritti nella Гоп́ $\epsilon \omega \nu ~ \varphi \omega ́ \varrho \alpha$, mentre considera forgiati forse dallo stesso Enomao la ventina di responsi oracolari a noi sconosciuti.

6 Oltre che dagli editori della Praeparatio Evangelica e dei frammenti della $\Gamma$ ó́ $\tau \omega \nu ~ \varphi \omega ́ \varrho \alpha$, il problema di Erodoto come fonte di Enomao non appare avvertito nello studio di Busine (2005: pp. 333-334) né in quello più recente di Addey (2014: pp. 109-110), che dedica alcune brevi pagine all'uso della Гó́ $\tau \omega v$ $\varphi \omega ́ \varrho \alpha$ di Enomao di Gadara nell'ambito della discussione sulla demonologia svolta dalla Praeparatio Evangelica di Eusebio.

7 In questa sede non si intendono affrontare, invece, le questioni riguardanti la storicità e la credibilità degli oracoli di Delfi le quali, oltre a calamitare l'attenzione degli interpreti, sono state oggetto di un numero notevolissimo di studi: sul tema si rinvia a Maurizio (1993; 1997); Kindt (2006); Gagné (2013). Sul problema dell'autenticità degli oracoli greci per il periodo tardoantico cfr. Bonnechere (2006); Busine (2014).

8 Il confronto dei frammenti di Enomao con i testi di Erodoto è, in qualche modo, limitato dalla perdita

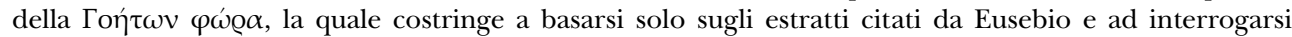
sulla effettiva fedeltà di quest'ultimo. Sulle numerose citazioni di Eusebio da autori antichi, gli studi dimostrano tuttavia che, soprattutto nella Praeparatio Evangelica, lo scrittore cristiano trasmette le sue fonti in modo perlopiù fedele, intervenendo non tanto attraverso delle modifiche dirette sul testo, quanto mediante l'attribuzione di interpretazioni tendenziose ai testi citati: cfr. Zink (1979: pp. 12-14); Inowlocki (2006); Zamagni (2010: pp. 57-82).

9 Parke \& Wormell $(1956 ; 1956 a)$.

10 In appendice al presente lavoro, si fornisce una tavola sinottica comprendente i testi degli oracoli di Delfi presi in esame, secondo le versioni corrispettive di Erodoto e di Enomao di Gadara. Il testo di Erodoto è riportato secondo l'edizione a cura di Colonna \& Bevilacqua (1996); per il testo problematico di Hdt. $5,92 \beta 2$ (PW6) - come si dirà infra - si seguono, invece, gli emendamenti al V libro di Erodoto presenti 
Un primo gruppo di sei riferimenti alle Storie di Erodoto è stato indicato da editori e commentatori dei frammenti di Enomao di Gadara in corrispondenza dei responsi oracolari di Apollo Pitico a Creso, re di Lidia. Essi sono tra i più celebri degli oracoli di Delfi, nonché emblema della caratteristica ambiguità espressiva che ha accompagnato l'oracolo nel corso di tutta la sua storia. Considerati perlopiù come privi di consistenza storica, tali responsi fanno parte della tradizione leggendaria su Creso riportata nel racconto di Erodoto, la quale appare organizzata in forma tragica sulla base di una possibile rielaborazione di fonti precedenti. ${ }^{11}$ I passi corrispondenti di Enomao, relativi a tre dei cinque responsi illustrati nelle Storie, non sono omogenei ma si distribuiscono in modo vario nell'ambito del virulento attacco al dio di Delfi per aver ingannato il supplice lidio, presto sconfitto dai Persiani.

Il primo ed il terzo oracolo di Creso, corrispondenti secondo la classificazione di Parke e Wormell a PW52 e PW54, sono citati solo in modo indiretto nella parte superstite del testo di Enomao preservata da Eusebio e, pertanto, non è possibile dire molto sulla forma in cui essi dovevano presentarsi nella fonte (o nelle fonti) consultata dall'autore

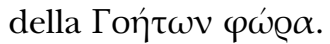

Nel caso di PW52, le allusioni di Enomao all'onniscienza di Apollo sono in forma di parodia e sbeffeggiano il dio ritorcendogli le stesse parole dell'oracolo. Il testo, che segna il passaggio dalla prima alla seconda persona singolare, è rielaborato e adattato all'apostrofe da Enomao, ma appare simile per struttura sintattica e lessicale ai versi riportati da Erodoto. ${ }^{12}$

nell'edizione a cura di Nenci (1994) per la Collana "Scrittori greci e latini" della Fondazione Lorenzo Valla (Milano: Arnoldo Mondadori Editore). Per i frammenti di Enomao di Gadara, si riporta l'edizione della Praeparatio Evangelica di Eusebio di Cesarea a cura di Mras (1954-1956 [1982²]). Le sigle utilizzate per designare gli oracoli di Delfi sono quelle del catalogo di Parke \& Wormell (1956a), ai quali si aggiunge parimenti il riferimento alle sigle corrispondenti del catalogo di Fontenrose (1978).

11 Sul problema delle fonti di Erodoto e dell'attendibilità del suo racconto, specie per la storia lidia ed orientale, si rimanda a Panitz (1935: pp. 45-49); Defradas (1954: pp. 208-228); Crahay (1956: pp. 182-207); Fehling (1989); Hartog (1980); Fowler (1996: pp. 62-87). Un utile prospetto sulla questione delle fonti di Erodoto, che è stato spesso accusato di inventare le proprie fonti e di travestire un'opera (pur notevole) di fantasia con i panni di una vera ricerca storica, è fornito da Luraghi (1994: pp. 181-190) e da Bettalli (2001: pp. 49-68). Sul ruolo degli oracoli delfici nel racconto erodoteo su Creso, Fontenrose (1978: p. 112) sostiene che essi assolvano ad una funzione letteraria e che siano da considerarsi come "steps in the story or drama, veiled warnings to the powerful and arrogant man and at the same time incitements to doom". In questo senso cfr. anche Barker (2006: pp. 1-28).

12 Cfr. Eus. p.e. 5, 21, 1, 1-3; 5, 34, 2, 1-2 (= Hdt. 1, 47). Identica al testo erodoteo appare la lezione

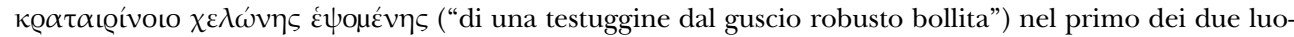
ghi di Enomao, che fa riferimento all'espediente adottato da Creso per verificare l'affidabilità dell'oracolo di Delfi. Nella letteratura greca, espressioni di onniscienza relative alla quantità dei granelli di sabbia o alle dimensioni del mare sono proverbiali: cfr. Hes. Op. 648; Pi. P. 9, 44-49. Secondo Rainart (2014: pp. 242-243), la seconda menzione dell'oracolo di PW52 nel testo di Enomao (Eus. p.e. 5, 34, 2, 1-2), ridotta ai soli primi due versi, suggerirebbe l'utilizzo di Plutarco (Plu. mor. 3.512e), il quale cita il verso 2

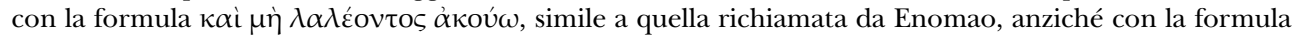

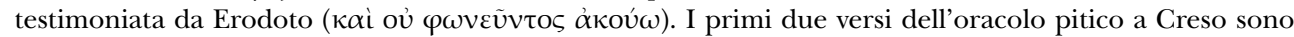
riportati da Eusebio anche nella più tarda Theophania, della quale rimangono solo pochi frammenti greci ed una versione siriaca: cfr. l'edizione a cura di Gressmann (1904: p. 111). 
Anche i passi relativi al terzo vaticinio (PW54), che Erodoto colloca dopo i doni e l'accordo di Creso con i Delfi, sembrano nel complesso aderenti alla versione dell'oracolo trasmessa dalle Storie, e riprendono l’immagine profetica del "mulo" (íníovos) che diventerà re dei Medi, allusione alla duplice estrazione sociale ed etnica - medo-persiana - di Ciro. ${ }^{13}$

Diverso è il caso del secondo responso delfico a Creso, corrispondente a PW53. Nelle Storie esso è richiamato da Erodoto in forma indiretta e annuncia, in quanto composizione chiaramente post eventum, la distruzione di un grande impero (che si rivelerà essere quello lidio) qualora Creso muova guerra ai Persiani. ${ }^{14}$ A differenza di Erodoto, due frammenti di Enomao riportano, in due distinti passi, un verso esametro relativo a tale responso pitico, che è trasmesso in forma diretta ed anche più completa rispetto alle Storie:

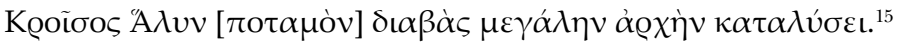

J. Fontenrose ha negato l'autenticità di tale versione e, contro l'opinione di $\mathrm{H}$. W. Parke e D. E. W. Wormell, ${ }^{16}$ ha sostenuto che il secondo oracolo delfico a Creso, alla pari del quinto (PW56 = Q103), avesse solo una forma indiretta nella tradizione orale, "though an occasional narrator might choose to express it in direct prose [...]". ${ }^{17}$ Tale considerazione non tiene sufficientemente conto di alcuni dati: il verso esametro di PW53 preservato da Enomao/Eusebio è tradito, nella medesima forma testuale, da una serie di autori di lingua greca a partire da Aristotele, il quale, nella Rhetorica, lo assume come esempio "didattico" nel contesto delle cinque regole sulla purezza retorica che respingono le ambiguità; ${ }^{18}$ il verso è noto anche a Cicerone, che nel De divinatione ne riproduce una traduzione in lingua latina; ${ }^{19}$ infine, alla versione ricordata da Enomao sembra riferirsi il contemporaneo Luciano di Samosata giacché quest'ultimo menziona, seppur in forma indiretta, l'attraversamento del fiume Halys come evento chiave nella realizzazione della profezia delfica, non riferito nel testo di Erodoto. ${ }^{20}$

Nessuno di questi autori richiama, inoltre, la seconda clausola del medesimo oracolo a Creso presente nella versione erodotea di PW53, in cui si consiglia al re di Lidia di allearsi con i più potenti tra i Greci. È verosimile che le ragioni di tale silenzio siano da riconoscersi nel fatto che questa clausola potrebbe aver costituito una seconda e distinta

13 Cfr. Eus. p.e. 5, 21, 4, 1-3 (= Hdt. 1, 55). Oltre alla forma indiretta, Enomao propone anche una citazione letterale dell'ultimo verso di PW54, testualmente conforme al passo di Erodoto, conservata in un successivo luogo eusebiano (Eus. p.e. 5, 24, 8, 7).

14 Hdt. 1, 53.

15 “Creso, attraversato il fiume Alys, distruggerà un grande impero" (trad. Migliore): cfr. Eus. p.e. 5, 20, 10, 9. Il verso è ripetuto da Enomao in ivi 5, 21, 2, 5 (privo però del soggetto).

16 Parke \& Wormell (1956: p. 133).

17 Fontenrose (1978: pp. 113-114).

18 Arist. rh. 3, 5, 4. Cfr. anche D.S. 9, 31, 1; Max. Tyr. 11, 2; Thdt. affect. 10, 139.

19 Cic. div. 2, 56, 115: Croesus Halyn penetrans magnam pervertet opum vim.

20 Luc. JConf. 14; JTr. 20 (43). 
risposta dell'oracolo data al momento della seconda consultazione,${ }^{21}$ la quale non necessariamente doveva essere conosciuta o citata di seguito alla prima dagli autori suindicati.

La trasmissione del verso esametro di PW53 conservato da Enomao e parimenti da altri autori (tra di loro indipendenti) sembra deporre a favore dell'esistenza, al di fuori di Erodoto, di una versione in esametri del secondo oracolo a Creso circolante in età antica, e di cui abbiamo traccia a partire dal IV sec. a.C.; completata da una seconda parte, nota solo in forma indiretta attraverso Erodoto, tale versione risulta essere espressa in terza persona singolare, in esatta conformità con quanto riferito nelle Storie, dato che l'oracolo si rivolgeva agli emissari di Creso e non direttamente al re lidio.

Riguardo ai presunti rapporti tra Erodoto ed Enomao, altri particolari problemi offrono i due oracoli agli Ateniesi, corrispondenti a PW94 e PW95. Si tratta dei due famosi responsi delfici enunciati in occasione dell'imminente invasione di Serse e riportati, nella narrazione di Erodoto, agli anni 481-480 a.C.; si è a lungo discusso sull'autenticità di tali pronunciamenti oracolari (al confine tra responsi storici e pseudo-storici) e, sebbene non si possa negare una forma di rielaborazione letteraria, sembrerebbe necessario riconoscere ad essi un nucleo di storicità, suggerito da vari elementi. ${ }^{22}$

Nella tradizione erodotea, i due oracoli agli Ateniesi sono composti ciascuno da dodici versi esametri. Riguardo a PW94, il testo di Erodoto, che costituisce il testimone più antico del responso delfico, ${ }^{23}$ appare rispecchiato fedelmente dalla versione confluita nella più tarda Anthologia Palatina ${ }^{24}$ e, relativamente al primo verso, da Giovanni Tzetzes, che ne attribuisce tuttavia la paternità al chresmologos Bakis, vissuto in un indistinto momento

21 L'ipotesi relativa ad una possibile articolazione di PW53 in due risposte successive appare suggerita dal riferimento di Erodoto al consenso dato ai Lidi rispettivamente da due oracoli, dopo la duplice interrogazione da parte degli emissari di Creso sulla convenienza di una campagna militare contro i Persiani

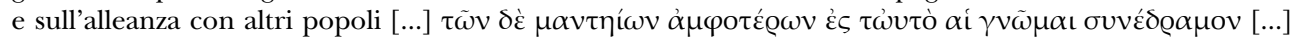
(Hdt. 1, 53, 3). A parere di Fontenrose (1978: p. 112), la seconda clausola di PW53 attestata nella versione erodotea potrebbe derivare da un responso autentico a Creso, al quale l'oracolo pitico poteva aver consigliato di allearsi con Sparta, la più forte delle poleis greche a quel tempo.

22 A giudizio di diversi studiosi, l'autenticità (almeno parziale) di PW94 e PW95 sarebbe sostenuta dalla vicinanza di Erodoto agli eventi del 480 o dall'interpretazione di Temistocle sul "muro di legno" come allusione alle navi, in accordo con la sua politica di potenziamento della flotta navale di Atene: cfr. Parke \& Wormell (1956: p. 170); Hammond (1959: p. 223); Hignett (1963: pp. 441-445). Per converso, elementi sospetti di artificiosità sono riconoscibili in altri aspetti, quali ad es. la lunga forma poetica e le modalità espressive dei due oracoli: cfr. Fontenrose (1978: pp. 124-128).

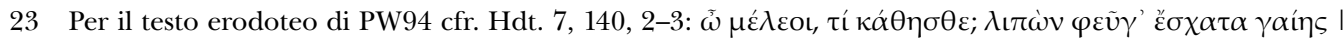

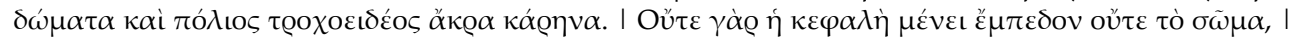

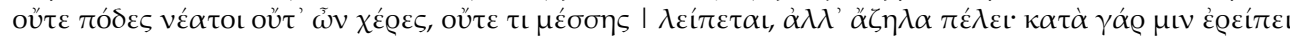

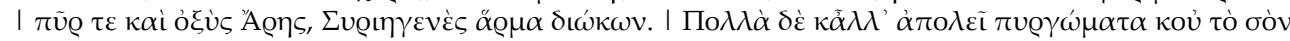

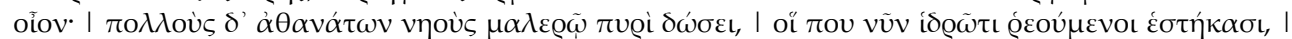

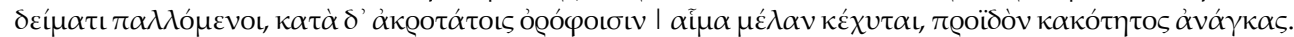

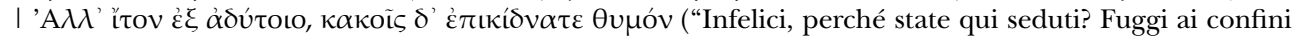
della terra, | abbandonando le case e le alte cime della città rotonda. | Né la testa resta salda né il corpo, | né i piedi né le mani; e niente di quell che vi è in mezzo | rimane, ma tutto è in uno stato miserando: la distruggono | il fuoco e Ares impetuoso, che guida un carro siriaco. | Abbatterà molte altre rocche, non solo la tua; | darà in preda al fuoco violento molti templi degli immortali, | che ora si ergono grondanti di sudore, | tremanti di spavento; e giù dagli altissimi tetti | nero sangue cola, presagio di sciagura inevitabile. | Ma uscite dal luogo sacro e dispiegate sulle sventure il vostro coraggio": trad. Colonna \& Bevilacqua). 
di età arcaica. ${ }^{25}$ Differente è la versione del responso pitico data da Enomao nel brano conservato da Eusebio, e riflessa in maniera incompleta da Teodoreto di Cirro, che dipende dal vescovo di Cesarea: ${ }^{26}$

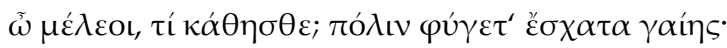

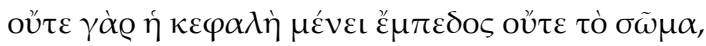

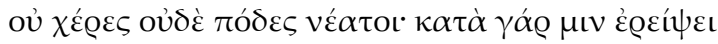

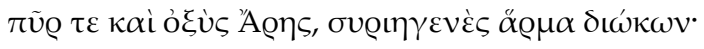

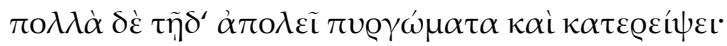

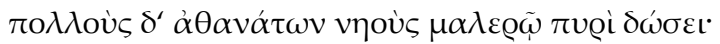

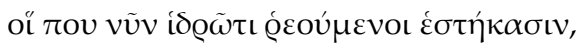
$\delta \varepsilon \dot{\mu} \mu \alpha \tau \iota \pi \alpha \lambda \lambda \operatorname{ó} \mu \varepsilon v o เ .^{27}$
\end{abstract}

Degli originali dodici versi attestati nelle Storie, il frammento di Enomao ne conserva solo otto e li incorpora in una versione più ridotta del responso delfico. Il brano tradito da Enomao/Eusebio non appare peraltro conforme testualmente ai versi di Erodoto, ma presenta variazioni di tipo morfo-sintattico e lessicale. Rispetto ad Erodoto, l'imperativo di pheugein ("fuggire") espresso dal dio al consultante, è svolto nel testo di Enomao/ Eusebio in seconda persona plurale anziché singolare. ${ }^{28}$ Nella versione di Erodoto, il participio aoristo attivo $\lambda \iota \pi \omega \omega v$, presente nel primo verso, si collega col verso immedia-

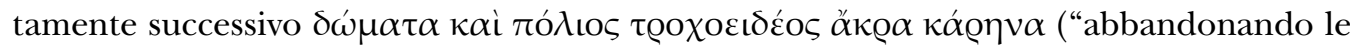
case e le alte cime della città rotonda”); diversamente, nel testo di Enomao il participio

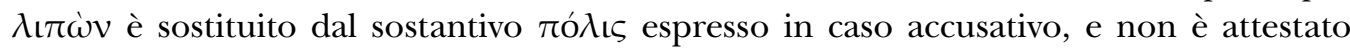
l'intero secondo verso, a cui esso era sintatticamente e logicamente connesso. Altre lacune individuabili nella versione fornita da Enomao riguardano l'abbandono e le tragiche conseguenze dell'incipiente devastazione, relative al quarto e quinto verso (oǔ $\tau \varepsilon$

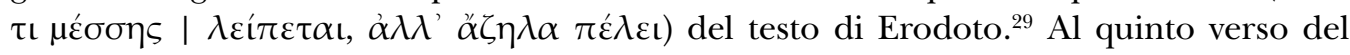

25 Jo. Tzet. chil. 9,812 .

26 Thdt. affect. 10, 140.

27 "O sciagurati, perché state fermi? Fuggite dalla vostra città fino ai confini della terra; I giacché non resta intatta la testa e neppure il corpo, | non le mani, né le estremità dei piedi; poiché tutto distruggerà | il fuoco e il terribile Ares, che guida il suo carro siriano; | distruggerà e abbatterà dalle fondamenta numerose mura turrite, | e consegnerà alla violenza del fuoco i templi degli immortali, | che già da ora, mentre ancora sono in piedi, grondano di sudore | e tremano per lo spavento" (trad. Migliore): cfr. Eus. p.e. 5, $24,1,4-11$.

28 In questo senso, la versione erodotea di PW94 si caratterizza per le sue "violent fluctuations in number between singular, dual and plural" (cfr. Parke \& Wormell 1956a: p. XXV) le quali, per via delle variazioni testuali e delle lacune, sono assenti nella versione tradita da Enomao. Sulla scelta indecisa tra il singolare ed il plurale dei verbi nella versione erodotea di PW94, cfr. Rainart (2014: pp. 100-101): "Ce va-et-vient entre le pluriel, le duel et le singulier donne le ton d'un oracle plein de conseils, qui prend le parti des Athéniens, tantôt comme s'ils étaient l'ensemble des citoyens de la grande cité, tantôt comme s'ils étaient liés par le sort que le destin réserve à chaucun d'eux et qui prend à partie les consultants. Par le dernier vers est donnée la preuve que les consultants sont au nombre de deux. [...] C'est un échange très vivant, sans doute l'exemple le plus évident de la vie réelle des oracles rendus à Delphes".

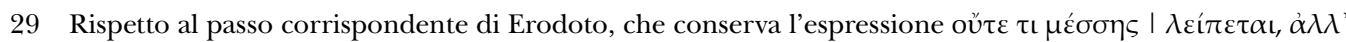

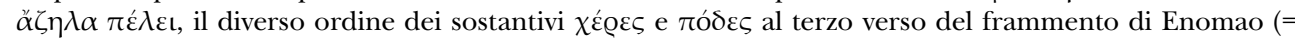




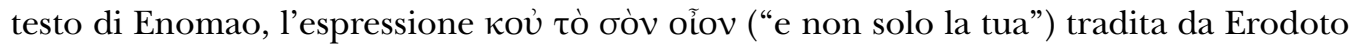

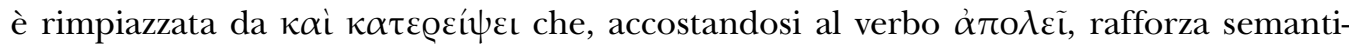
camente il concetto della forza demolitrice di Ares. ${ }^{30}$ La versione del testo di Enomao appare, inoltre, decurtata o comunque incompleta, interrompendosi a metà del decimo verso del brano di Erodoto: non c'è traccia, infatti, degli ultimi versi relativi all'immagine

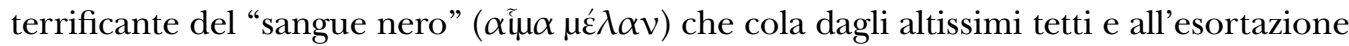
finale ad uscire dall'adyton indirizzata agli Ateniesi. ${ }^{31}$

Divergenze testuali sono rintracciabili parimenti nel secondo responso agli Ateniesi (PW95), tornati in un momento consecutivo con rami di supplici, dietro consiglio del delfo Timone, a consultare l'oracolo pitico. La versione delle Storie erodotee, costituita da dodici versi, ${ }^{32}$ anche in questo caso corrisponde nella sua interezza a quella consegnata dall'Anthologia Palatina,${ }^{33}$ nonché ad un verso trascritto da Luciano, ${ }^{34}$ che è rimasto invariato nella tradizione di Enomao/Eusebio. Quest'ultima, citata in modo incompleto da Teodoreto attraverso Eusebio, ${ }^{35}$ differisce tuttavia notevolmente dai passi riportati da Erodoto:

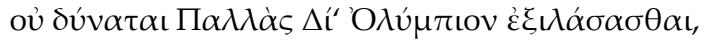

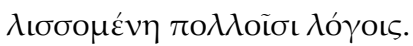

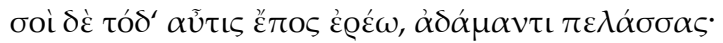

$\tau \tilde{\omega} \nu \not \ddot{\alpha} \lambda \lambda \omega \nu \pi \varrho 0 \alpha \lambda \iota \sigma \kappa о \mu \varepsilon \dot{v} \omega \nu$

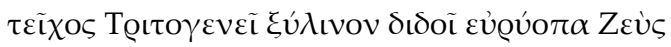

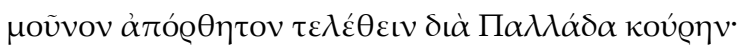

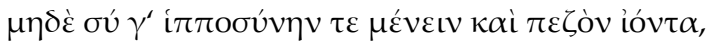

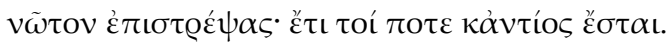

Eus. p.e. 5, 24, 6) è, probabilmente, dovuto a ragioni metriche.

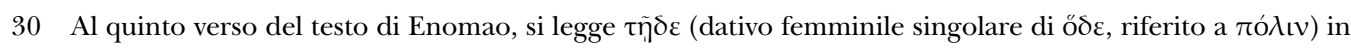

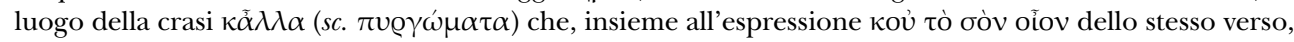
esprime il concetto della distruzione di altre rocche nella versione di Erodoto.

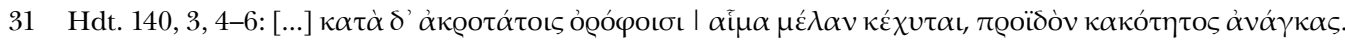

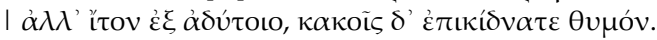

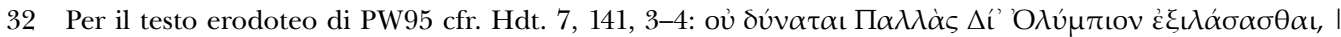

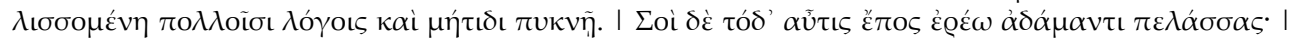

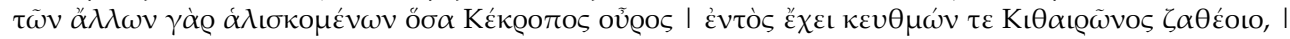

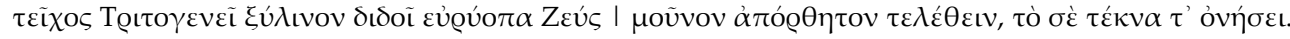

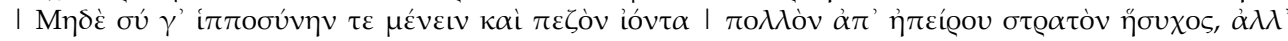

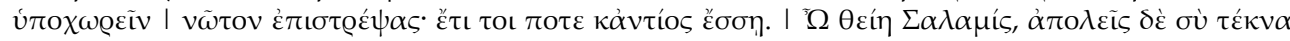

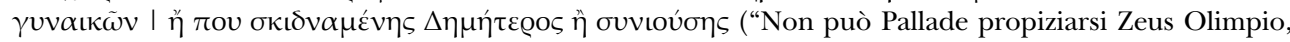
| pur pregandolo con molte parole e con accorta saggezza; | ma a te dirò ancora quest'altro responso, rendendolo saldo come l'acciaio. | Quando sarà preso tutto ciò che racchiudono il monte di Cecrope | e i recessi del Citerone divino, Zeus dall'ampio sguardo concede alla Tritogenia che rimanga inviolato । il muro di legno soltanto, che te salverà e i tuoi figli. | E tu non aspettare tranquillo la cavalleria e la fanteria che avanza | in massa dal continente, ma ritirati | volgendo le spalle: giorno verrà in cui sarai con il nemico fronte a fronte. | O divina Salamina, farai perire figli di donne | o quando si semina o quando si raccoglie il frutto di Demetra": trad. Colonna \& Bevilacqua).

APl. 14, 93.

34 Luc. JTr. 20: $\tilde{\omega} \theta \varepsilon i ́ \eta \eta$

Thdt. affect. 10, 140. 


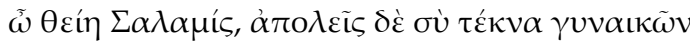

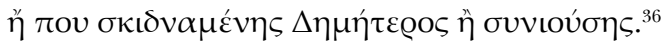

Anche qui il testo di Enomao risulta essere più breve rispetto alla versione erodotea, e non è esente da cambiamenti a livello sintattico e lessicale. Rispetto al relativo esametro di Erodoto, il secondo verso del testo di Enomao si arresta in corrispondenza della

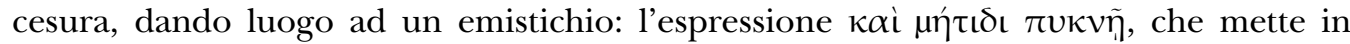
evidenza la nozione della $\mu \tilde{\eta} \tau \iota \varsigma$ di Pallade, non è infatti riportata nel testo dell'oracolo trasmesso da Enomao. Un'ulteriore e ben più ampia lacuna nella versione di PW95 della Гớ $\omega \omega v \varphi \omega ́ \mathrm{Q} \alpha$ riguarda, poi, parte del quarto e l'intero quinto verso della versio-

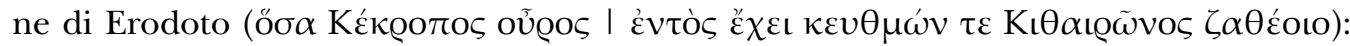
del quarto verso esametro rimane, nel testo di Enomao, solo il genitivo assoluto ( $\tau \tilde{\omega} v$ $\not \alpha \lambda \lambda \omega \nu \pi \varrho 0 \alpha \lambda \iota \sigma \kappa о \mu \varepsilon ́ v \omega \nu)$, che costituisce un emistichio da un punto di vista metrico; in luogo del verbo $\alpha \lambda \tilde{\omega} v \alpha \iota$ è attestato il verbo composto $\pi \varrho o \alpha \lambda \hat{\omega} v \alpha$, nella for-

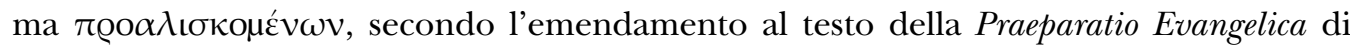

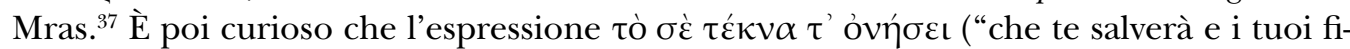

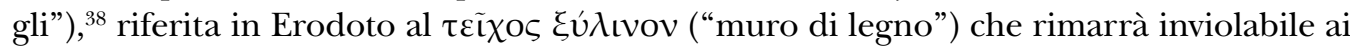

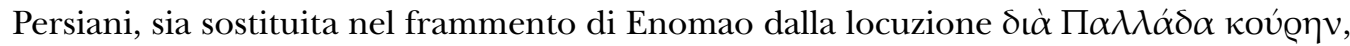
che costituisce un pleonasmo, giacché il riferimento all'intercessione di Atena Tritogenia presso Zeus a favore degli Ateniesi è già contestualmente e sintatticamente esplicitato. Del tutto assente nella versione di Enomao è, inoltre, il nono verso del testo dell'oracolo

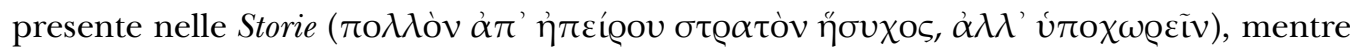

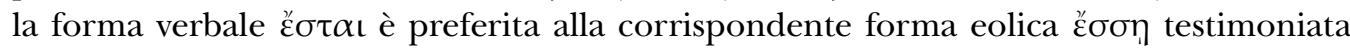
da Erodoto. Maggiore sconcerto suscita la versione di PW95 fornita da Clemente Alessandrino. L'estratto citato dallo scrittore cristiano negli Stromata riporta infatti in uno stesso luogo solo alcuni versi, estrapolati da ambedue i responsi delfici agli Ateniesi, e ne inverte l'ordine. ${ }^{39}$ Cambia così, nel brano di Clemente, il soggetto della frase, dando

36 "Pallade non può placare Zeus Olimpio, | pur scongiurandolo con ripetute suppliche. | Ma a te ancora dirò questa parola, inalterabile come l'acciaio: | quando le altre città saranno prese | alla Tritogenia Zeus onniveggente concede che solo un muro | di legno sia inespugnabile per amore della vergine Pallade. | E tu non attendere la cavalleria e la fanteria che avanza, | ma, volgendo le spalle, [ritirati]: un giorno avrai ancora di fronte il nemico. | O divina Salamina, tu farai perire figli di donne, | o quando Demetra semini, o quando raccolga il frutto" (trad. Migliore): cfr. Eus. p.e. 5, 24, 2, 5-14.

37 Cfr. Mras (1954: p. 266). I codd. della Praeparatio Evangelica di Eusebio riportano variamente la lezione

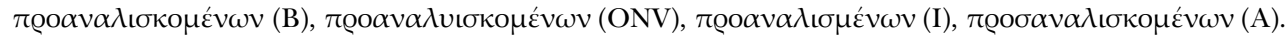

38 Hdt. 7, 141, 3, 7.

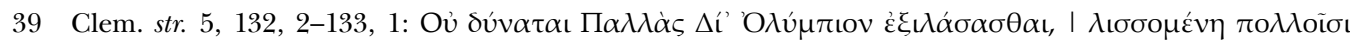

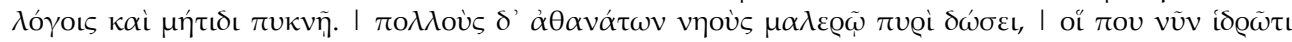

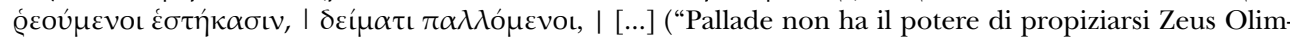
pio, pur pregandolo con molte parole e senno profondo; ma egli darà in preda a fuoco vorace molti templi degli immortali, [le cui statue] ora si ergono grondanti sudore, tremanti di spavento etc.": trad. Pini). L'estratto di Clemente è una combinazione dei primi due versi di PW95 (= Hdt. 7, 141, 3, 1-2) seguiti dai versi alle linee 8-9-10 di PW94 (= Hdt. 7, 140, 3, 2-3-4) secondo il testo di Erodoto, i quali sono testualmente corrispondenti alla versione fornita dalle Storie. Oltre alla tradizione diretta, il brano di Clemente è riportato anche dalla Praeparatio Evangelica di Eusebio, nonostante essa citi già in alto le versioni di Enomao sui due oracoli agli Ateniesi: cfr. Eus. p.e. 13, 13, 61, 5-6 e 7-9. 
luogo ad un vero nonsense: artefice degli incendi che saranno appiccati ai templi greci

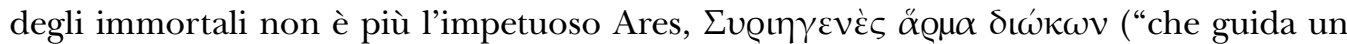
carro siriaco”), chiara allusione a Serse, ma nientemeno che Pallade (sic!). ${ }^{40}$

Si delineano in tal modo tre versioni dei due oracoli delfici agli Ateniesi, risalenti rispettivamente a Erodoto, Enomao di Gadara e Clemente Alessandrino (almeno nella loro tradizione più antica a noi conservata), le quali impongono una serie di necessari interrogativi. Tali autori riflettono in effetti tre distinti modelli testuali, relativi ai due oracoli delfici agli Ateniesi, disponibili in antico? Se non genuine, le variazioni riscontrate nelle due versioni di Enomao/Eusebio e di Clemente costituiscono delle manomissioni ad opera degli stessi autori (o delle loro fonti) o sono dovute ad un'eventuale corruzione della tradizione manoscritta delle loro opere? Non c'è dubbio che Erodoto restituisca una versione qualitativamente migliore di tali testi oracolari, che sembra essere preferita ad ogni altra da vari autori ancora in età imperiale e bizantina: le Storie dovevano infatti rappresentare, per la loro antichità, un testo "classico" nell'ambito della documentazione sulle guerre persiane. Il tipo di discrepanze testuali individuabili presso i tre autori in questione sembra suggerire l'utilizzo di tre fonti (e dunque di tre versioni) differenti e tra loro indipendenti dei due oracoli agli Ateniesi, verosimilmente disponibili nel mondo antico nell'ambito della tradizione letteraria sugli oracoli delfici. La possibilità di interventi individuali ad opera di Enomao o di Clemente appare inoltre improbabile, dal momento che entrambi polemizzavano (da punti di vista differenti) con l'oracolistica dei pagani ed avevano interesse a citare correttamente i documenti riguardanti gli avversari.

Delle osservazioni significative offre parimenti il testo dell'oracolo delfico a Licurgo corrispondente a PW29. Parte della celebre tradizione "leggendaria" sull'origine della costituzione di Sparta, esso rappresenta le parole con cui la Pizia dà il benvenuto a Licurgo, al momento del suo ingresso nel tempio di Apollo, esprimendo l'incertezza se considerarlo come dio o come uomo. L'oracolo, che sancisce il carattere divino di Licurgo, ${ }^{41}$ è trasmesso in una versione di quattro versi esametri da Erodoto ${ }^{42}$ e lo stesso testo è prodotto, in forma completa e incompleta, da altri testimoni di epoca successiva. ${ }^{43}$

40 Sull'approccio di Clemente ai testi oracolari attribuiti ad Apollo, a cui egli riconosce un valore profetico in termini di verità cristiana, cfr. Sardella (1988: pp. 295-329); Busine (2005: pp. 369-373).

41 Secondo Meyer (1892: pp. 269-283), tale oracolo rappresenterebbe la sanzione delfica del culto di Licurgo come dio in Sparta, dove gli era stato dedicato un santuario e gli erano tributati sacrifici annuali. A parere di Fontenrose (1978: p. 115), il culto spartano di Licurgo sarebbe probabilmente da antedatarsi rispetto all'istituzione oracolare di Delfi.

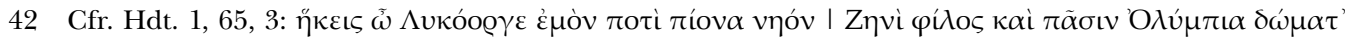

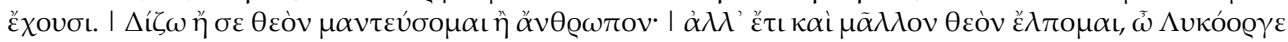
("Sei giunto, o Licurgo, al mio ricco tempio, | tu caro a Zeus e a quanti abitano le dimore dell'Olimpo. | Non so se proclamarti un dio o un uomo; | ma inclino a pensare che tu sia un dio, o Licurgo": trad. Colonna \& Bevilacqua).

43 Gal. Protr. 9, 22; Them. Or. 7, 97d; 15, 193c; 19, 225d. In forma indiretta vi si riferiscono Val. Max. 5, 3 ext. 2; Philostr. VA 8, 7. La medesima versione di PW29 fornita da Erodoto è attestata dal primo dei due brani relativi all'oracolo pitico riportati dalla Anthologia Palatina (14, 69). Sebbene vi alluda perlopiù in forma indiretta, la citazione di PW29 fatta da Senofonte, relativamente all'unico verso da lui trasmesso verbatim

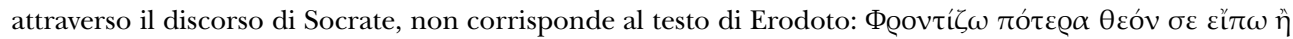

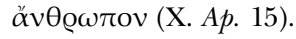


Nella Praeparatio Evangelica, il testo dell'oracolo è riferito direttamente da Eusebio nel commento introduttivo al successivo frammento di Enomao ed al suo ammonimento dei responsi rivelati al legislatore spartano. È, tuttavia, fuor di dubbio che il testo trascritto da Eusebio appartenga ad una sequenza perduta della Гoฑ́ $\tau \omega \nu ~ \varphi \omega ́ \varrho \alpha$, data l'attitudine del vescovo di Cesarea ad anticipare la materia di dibattito degli excerpta di Enomao, come avviene nel caso dei responsi ad Archiloco, ad Euripide e ad Omero, preceduti dalla discussione dello scrittore cristiano. ${ }^{44}$ Il testo di Enomao/Eusebio, espresso come gli altri testimonia in seconda persona singolare, si compone di sei versi:

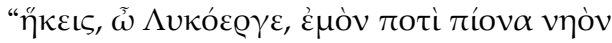

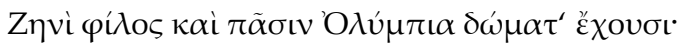

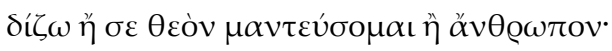

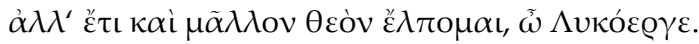

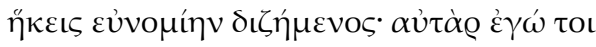
$\delta \omega \omega \sigma \omega$
$[\ldots] "{ }^{45}$

Il brano appare identico a quello corrispondente di Erodoto, ad eccezione degli ultimi

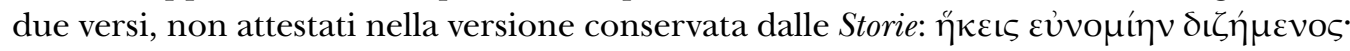

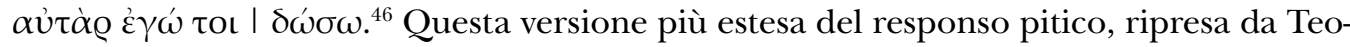
doreto di Cirro che dipende da Eusebio, ${ }^{47}$ si confronta bene con Diodoro Siculo.

In uno degli Excerpta Vaticana del libro VII della Biblioteca storica si legge, infatti, una versione dell'oracolo a Licurgo completa di due versi finali, i quali pur differiscono

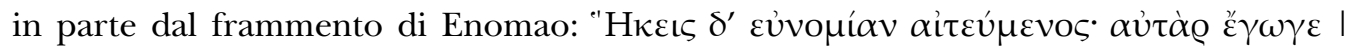

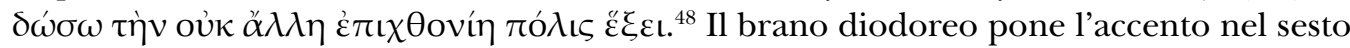
verso (rimasto incompleto nel testo di Enomao/Eusebio) sull'unicità della concessione fatta da Apollo a Sparta, giacché il dio darà ad essa la migliore costituzione mai avuta dalle altre città. I due versi finali testimoniati da Diodoro e da Enomao e riecheggiati da

44 Eus. p.e. 5, 32, 2; 33, 1-3. In generale, commentatori ed editori rispettivamente dei testi di Enomao, di Eusebio e degli oracoli di Delfi considerano il brano della Praeparatio Evangelica relativo a questo responso a Licurgo come un frammento appartenente alla perduta Гṓ $\omega \omega \nu \varphi \omega ́ \alpha$ di Enomao di Gadara. In ogni modo, il passaggio dell'oracolo che a noi interessa è ripetuto all'interno del frammento di Enomao citato da Eusebio, il che dissolve ogni dubbio sulla sua provenienza: cfr. Eus. p.e. 5, 28, 2, 7-8.

45 "Sei giunto nel mio ricco tempio, o Licurgo, | tu caro a Zeus e a quanti abitano le dimore dell'Olimpo; | sono in dubbio se proclamarti dio o uomo. | Ma spero piuttosto che tu sia un dio, o Licurgo. | Tu vieni a chiedermi una buona legislazione; ebbene io te la darò" (trad. Migliore): cfr. Eus. p.e. 5, 27, 8, 5-8.

46 Come già detto supra, la trasmissione di questi due versi è confermata dalla citazione letterale dell'estratto di Enomao, il quale schernisce la risposta alquanto vaga data a Licurgo da Apollo Pitico: Eus. p.e. 5, 28, 2, 7-8.

47 Thdt. affect. 10, 140-141. La versione di sei versi di PW29 (= PW216) trascritta da Arsenio Apostolio (8, 46a) deriverebbe, secondo Fontenrose (1978: p. 270), da Eusebio o da Teodoreto.

48 "Thou com'st in search of goodly laws; and such | a system of fair laws shall I now give | to thee as never city upon earth I shall e'er possess" (trad. Oldfather): cfr. D.S. 7, 12, 1 (Excerpta Vatic.). Nel catalogo di Parke e Wormell, i distinti passi di Diodoro e di Enomao, benché tra di loro parzialmente differenti, corrispondono a PW216. 
Plutarco, ${ }^{49}$ sono richiamati, con notevoli difformità, anche nel secondo dei due brani della Anthologia Palatina sull'oracolo pitico a Licurgo, che presenta una veste letteraria

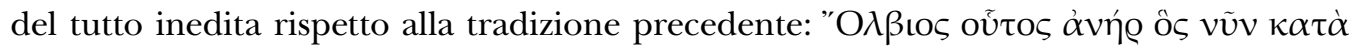

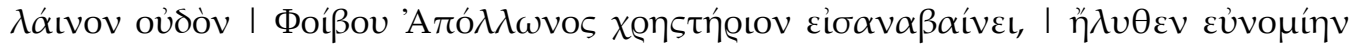

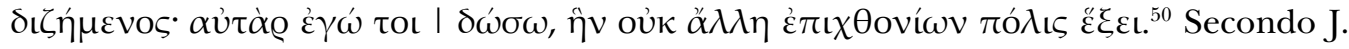
Fontenrose, la mancanza nella versione erodotea di PW29 di una risposta effettiva a Licurgo potrebbe aver indotto qualcuno ad aggiungere, in tempi successivi, i due esametri finali riportati da Diodoro. ${ }^{51}$ Questa ipotesi appare difficilmente ammissibile alla luce della pluralità e dell'interdipendenza di versioni estese dell'oracolo a Licurgo (Diodoro Siculo, Enomao, Anthologia Palatina), tra di esse denotate da numerose varianti; lo stesso Erodoto non può inoltre considerarsi all'origine della tradizione su Licurgo o suo unico informante, giacché in età antica, al di fuori delle Storie, potevano essere consultati ben altri documenti e fonti letterarie sulla tradizione riguardante il legislatore e la riforma costituzionale di Sparta.

C'è da credere che Diodoro Siculo, e come lui Enomao, abbia utilizzato una tradizione diversa dalle Storie di Erodoto. Nel successivo passaggio dello stesso frammento diodoreo viene riportato un secondo oracolo delfico a Licurgo (PW218) che, in una consultazione separata, sanziona la riforma costituzionale di Sparta, suggerendo i principi ai quali essa dovrà ispirarsi. ${ }^{52} \mathrm{Il}$ medesimo testo dato da Diodoro si trova anche in uno degli estratti di Enomao citati nella Praeparatio Evangelica:

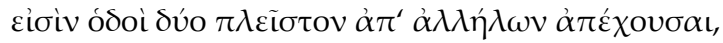

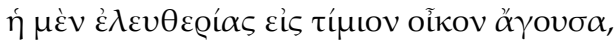

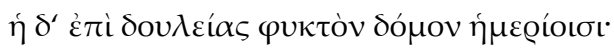

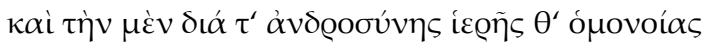

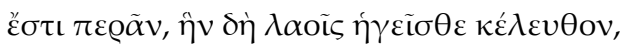

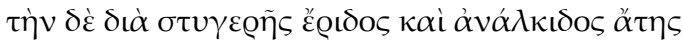

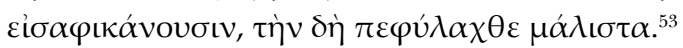

I versi oracolari indirizzati all'aspirante legislatore, cui è consigliato di guidare il popolo lungo la strada che conduce alla libertà ( $\dot{\varepsilon} \lambda \varepsilon v \theta \varepsilon @ i ́ \alpha)$ evitando la rotta della schiavitù

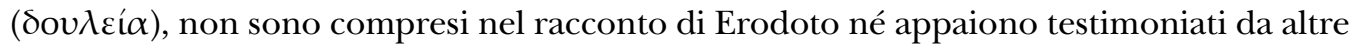
fonti al di fuori di Diodoro e di Enomao, allo stato attuale della documentazione.

49 Plu. Lyc. 5, 3.

50 "Fortunato quest'uomo, che qui sulla soglia di pietra | entra nel mantico tempio di Febo Apollo. Una giusta | costituzione richiede, che io gli darò volentieri: | nessun'altra città ne avrà mai l'uguale nel mondo" (trad. Pontani): cfr. APl. 14, 77.

51 Fontenrose (1978: p. 116).

52 D.S. 7, 12, 2 (Excerpta Vatic.).

53 "Esistono due strade molto distanti l'una dall'altra: | una conduce al venerato domicilio della libertà, | l'altra conduce alla casa della schiavitù, che i mortali devono fuggire. | L'una si percorre col coraggio e la divina armonia; | è la strada che devi mostrare ai popoli. | All'altra si giunge con la penosa discordia e la terribile miseria: è questa che bisogna guardarsi dal prendere" (trad. Migliore): cfr. Eus. p.e. 5, 28, 7, 1-7. 
Questo responso della Pizia restituisce un tassello mancante relativo all'origine "delfica" della riforma licurghea, soffermandosi sull'ispirazione, che è profetica ed etica insieme, attribuita all'oracolo. Tale momento della consultazione di Licurgo, precedente alla concessione della Rhetra, non viene particolarmente discusso nemmeno da Plutarco, che pure nella Vita Lycurgi fornisce la versione più ampia e dettagliata che ci sia giunta sulla derivazione pitica della riforma licurghea. Secondo la narrazione plutarchea, ad Apollo Pitico risalirebbe l'origine della Rhetra, la quale prescrive a Licurgo la realizzazione di un tempio a Zeus Sillanio e ad Atena Sillania, la suddivisione del damos in phylai e obai, la costituzione della gherousia, dei due archagetai e dell'assemblea dei cittadini, l'apella, la quale deve riunirsi periodicamente tra Babica e Cnacione $;^{54}$ successiva sarebbe, invece, l'appendice dei basileis Polidoro e Teopompo, anch'essa avallata secondo Plutarco dall'oracolo di Delfi, come indicherebbe un frammento di Tirteo citato verbatim dal filosofo di Cheronea ${ }^{55}$ Le notizie sull'ordinamento politico e legislativo di Sparta - le quali sono state al centro di una discussione interminabile tra gli interpreti circa la loro datazione e paternità - non appaiono menzionate da Erodoto e, diversamente, sono state oggetto in epoca greca e romana di una quantità di trattazioni teoriche, tra le quali la Costituzione di Sparta di Senofonte, alla quale Plutarco si rifà più volte nel corso della sua biografia. ${ }^{56}$

Come bisogna dunque giudicare le tradizioni sull'oracolo di Licurgo trasmesse da Erodoto e da Enomao? Dall'analisi sembra emergere una fisionomia divergente di esse per natura e provenienza. Nel resoconto delle Storie, Erodoto non sembra interessarsi né si sofferma particolarmente su Licurgo e sul ciclo di leggende e di oracoli che lo riguardavano. Egli tramanda brevemente il ricordo dell'origine "pitica" della riforma licurghea (lasciandolo precedere da una citazione di quattro versi dell'oracolo PW29) e menziona un'altra tradizione, di origine spartana, secondo la quale Licurgo avrebbe introdotto da Creta la sua riforma legislativa, al tempo in cui era tutore di Leobote, re di Sparta. ${ }^{57}$

Enomao sembra invece attingere ad una più particolareggiata tradizione di "tendenza" delfica, la quale riconduceva ad Apollo Pitico l'origine della Rhetra introdotta da Licurgo: nella sua polemica antioracolare, il filosofo cinico testimonia importanti

54 Plu. Lyc. 6, 2-6. Nonostante la polisemia del termine @́ít@ $\alpha$, traducibile con "patto, cosa detta", ma anche con "trattato", "decreto/legge" o "proposta", per Plutarco esso designa un oracolo (manteia), probabilmente perché egli riconosceva un'origine divina di tali leggi: cfr. Meriani \& Giannattasio Andria (1998: p. 40, n. 44$)$.

55 Sulla prescrizione di Polidoro e Teopompo alla Rhetra licurghea, che concedeva alla gherousia il diritto di veto sugli emendamenti legislativi dell'apella ritenuti eccessivi, cfr. Plu. Lyc. 6, 7-9, che conserva Tyrt. fr. $\mathrm{I}^{\mathrm{b}}$ (Gentili \& Prato 1979).

56 Sulle fonti riguardanti la legislazione di Sparta e la sua origine, è lo stesso Plutarco a nominare alcune

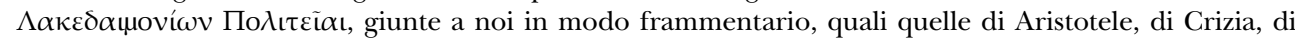
Dioscoride e di Sfero: Plu. Lyc. 1, 2; 5, 12; 6, 4; 9, 7; 11, 9; 28, 2; 28, 7; 28, 11; 31, 4. Oltre a tali opere monografiche, passaggi riguardanti particolari della vita di Licurgo o delle istituzioni spartane erano disseminati nei testi di vari autori, talvolta citati da Plutarco (e.g. Eratostene, Apollodoro di Atene, Timeo di Tauromenio, Simonide di Ceo, Dieutichida, Aristocrate di Sparta, Sosibio di Sparta): cfr. in merito Meriani \& Giannattasio Andria (1998: pp. 14-15).

57 Hdt. 1, 65, 4-5. Secondo Fontenrose (1978: p. 271), le due opinioni riportate da Erodoto sulla derivazione della riforma di Licurgo non sarebbero incompatibili, in quanto la Pizia potrebbe avere solo sanzionato le riforme costituzionali, senza dettarle. 
passaggi relativi alla genesi della riforma spartana ed è il solo a conservare, oltre ad una delle versioni estese di PW29 (= PW216), due oracoli inediti a Licurgo che precedono la "rivelazione" apollinea della Rhetra, uno dei quali è riportato anche da Diodoro Siculo. ${ }^{58}$

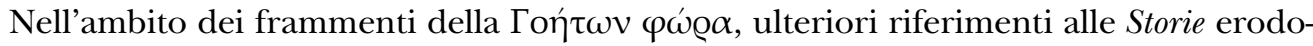
tee sono indicati da editori e commentatori in corrispondenza di altri cinque oracoli di Delfi, afferenti a vicende e personaggi svariati del mondo greco. Nell'ordine stabilito nel testo preservato dalla Praeparatio Evangelica, si tratta dei seguenti responsi pitici:

- PW100: l'oracolo agli Spartani, pronunciato secondo il racconto di Erodoto al principio della seconda guerra persiana, è composto nella versione testuale attestata dalle Storie e dalla Anthologia Palatina da sette esametri, ${ }^{59}$ dei quali il testo citato da Enomao trasmette solo i primi quattro. ${ }^{60}$

- PW63: il responso agli Cnidi, con cui il dio ammonisce il progetto di realizzazione di un canale che possa difendere Cnido dall'assalto del medo Arpago (545 a.C.), è trasmesso in identica forma, nei suoi due soli trimetri giambici, da Erodoto, Enomao e dalla Anthologia Palatina. ${ }^{61}$

- PW6 e PW8: si tratta rispettivamente dei due oracoli ad Eezione e a suo figlio Cipselo, tiranno di Corinto, che figurano nelle Storie di Erodoto all'interno del discorso del corinzio Sosikles. ${ }^{62}$ Caratterizzati da formule ed artifici tipici degli oracoli leggendari, questi testi appaiono incompleti degli ultimi versi nei frammenti della Гoฑ́ $\omega \omega \nu \varphi \omega \varrho \alpha^{63}$ rispetto alla versione fornita da Erodoto e dall'Anthologia Palatina. ${ }^{64}$ Un particolare problema filologico è poi riscontrabile nel caso di PW6, che nella citazione riportata nel

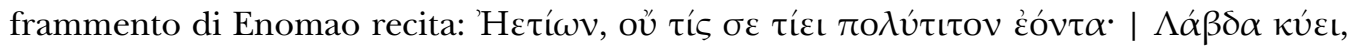

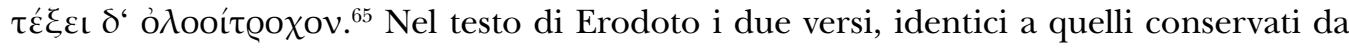
Enomao/Eusebio ed anche dall'Anthologia Palatina, sono stati emendati a ragione da G.

58 I due oracoli in questione corrispondono, nella classificazione di Parke e Wormell a PW220, su cui cfr. Eus. p.e. 5, 28, 3, 3-8, e al già discusso PW218. In uno dei frammenti dal settimo libro della Biblioteca storica, Diodoro trasmette un altro oracolo delfico a Licurgo (PW222), non presente tra i frammenti conservati di Enomao, relativo all'avarizia e alla sciagura che colpirà Sparta se contaminata dall'amore per il denaro: D.S. 7, 12, 5. Altri testimoni indicano come consultanti di PW222, invece, gli Spartani o i re Alcomene e Teopompo: cfr. Fontenrose (1978: p. 272).

59 Hdt. 7, 220, 4; APl. 14, 96. Tale oracolo di Apollo agli Spartani, che nelle Storie erodotee appare determinante nella strategia scelta da Leonida e nel massacro alle Termopili, è generalmente considerato come un vaticinio ex eventu: cfr. Parke \& Wormell (1956: pp. 167-168); Parke \& Wormell (1956a: p. 44); Fontenrose (1978: pp. 77-78). Secondo Legrand (1951: pp. 189-190), l'oracolo agli Spartani si proponeva lo scopo di giustificare la condotta di Leonida agli occhi di chi considerava un sacrificio inutile e sconsiderato la decisione di rimanere alle Termopili a prezzo della propria vita e di quella di trecento uomini scelti.

60 Eus. p.e. 5, 25, 2, 3-6.

61 Hdt. 1, 174; APl. 14, 81. Per il frammento di Enomao cfr. Eus. p.e. 5, 26, 2, 2-3.

62 Hdt. 5, $92 \beta 2$ (PW6); 5, 92 \& 2 (PW8).

63 Eus. p.e. 5, 29, 2, 2-3 (PW6); 5, 35, 1, 1-2 (PW8). A proposito dell'oracolo a Cipselo, vi è una corruzione nel testo di Enomao conservato da Eusebio, il quale riporta la lezione Kú $\psi \varepsilon \lambda$ os Ai $\alpha \kappa i ́ \delta \eta \varsigma$ anziché

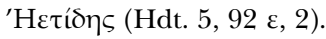

64 APl. 14, 86 (PW6); 14, 88 (PW8).

65 “[...] O Eezione, nessuno ti onora, pur essendo degno di molto onore; I Labda è incinta, genererà un macigno rotondo" (trad. Migliore). 


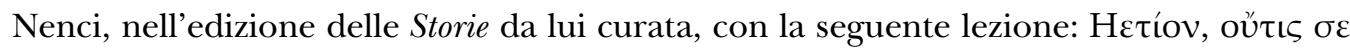

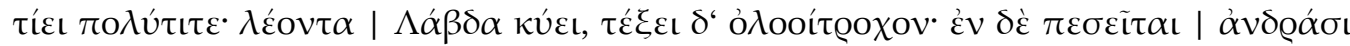

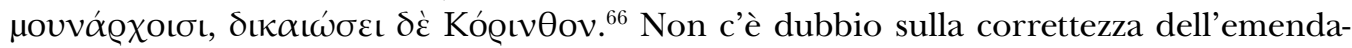

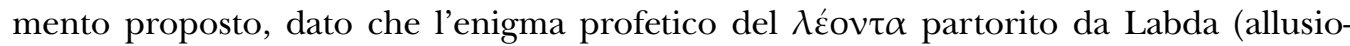
ne a Cipselo) non solo compare ma è anche il punto focale dell'oracolo ai Bacchiadi (PW7). ${ }^{67}$ Sulla base del confronto con le versioni di Enomao e dell'Anthologia Palatina,

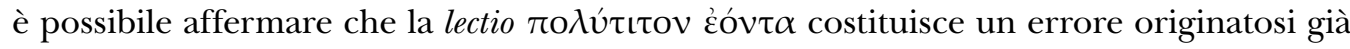
in età antica nella tradizione letteraria dell'oracolo PW6, e non appare ascrivibile ad una ipotetica corruzione della tradizione manoscritta di età medievale delle Storie, della Praeparatio Evangelica o della Anthologia Palatina.

- PW92: la breve citazione di Enomao del responso pitico agli Argivi, ${ }^{68}$ ai quali la Pizia raccomanda di non entrare in guerra con i Persiani (481 a.C.), è incompleta dell'ultimo verso rispetto alla versione tradita da Erodoto e dalla Anthologia Palatina. ${ }^{69}$

I testi degli oracoli delfici citati nei frammenti in questione della Гoฑ́ $\omega \nu \varphi \omega ́ \varrho \alpha$ appaiono complessivamente uguali alle versioni conservate nelle Storie di Erodoto e, in ogni modo, non recano le variazioni formali e le difformità individuate nei casi precedentemente osservati. Ci si può chiedere se l'assenza degli ultimi versi che connota i corrispondenti brani di Enomao di PW6, PW8, PW92 e PW100 sia dovuta alla status testuale delle versioni a lui disponibili o, come sembra possibile, ad una operazione di cutting determinata da necessità di sintesi: nei relativi contesti, il filosofo sta infatti rapidamente scorrendo con fini polemici alcuni degli oracoli pagani (nel tentativo, ad esempio, di svelare l'inutilità di alcune delle rivelazioni profetiche o l'adulazione dei governanti da parte dell'oracolo delfico) e ciò potrebbe averlo indotto a selezionare solo quello che, nei singoli casi, era più utile alle sue argomentazioni.

$$
* * *
$$

Posti i limiti derivabili dalla varietà di interventi testuali cui appare soggetto il fenomeno letterario della citazione e il più generale processo della tradizione manoscritta dei testi antichi, ${ }^{70}$ è bene tracciare un bilancio finale relativo alla comparazione delle versioni degli oracoli delfici trasmesse rispettivamente da Erodoto e dai frammenti di Enomao di Gadara conservati nella Praeparatio Evangelica di Eusebio.

Dal punto di vista testuale, le Storie erodotee restituiscono una versione degli oracoli di Delfi qualitativamente migliore rispetto ai brani corrispondenti di Enomao/Eusebio, dimostrando accuratezza nella scelta della versione più adatta e artisticamente più

66 "Eezione, nessuno onora te, o degno di molto onore; un leone | Labda ha nel ventre ma lo partorirà come un macigno precipite. | Poi si abbatterà sugli uomini che regnano da soli e punirà Corinto": cfr. Nenci (1994: pp. 102, 289-290).

67 Hdt. 5, 92 ß 3 (PW7).

68 Eus. p.e. $6,7,4,3-4$.

69 Hdt. 7, 148, 3; APl. 14, 94.

70 Oltre al processo di trasmissione testuale, va rilevata la questione della dimensione orale nella tradizione dei testi degli oracoli: sul tema cfr. Nagy (1996). 


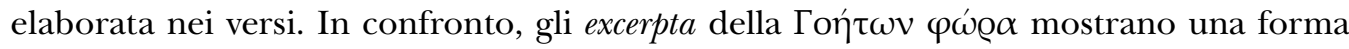
testuale dissimile e non sempre di pari livello letterario.

Lo studio analitico degli estratti dei due autori condotto attraverso il controllo incrociato con i vari testimonia che compongono la documentazione superstite riguardante gli oracoli delfici esaminati consente di ricavare ulteriori dati.

Secondo quanto emerso dall'analisi, appare improbabile la possibilità che Enomao abbia consultato e ritoccato le versioni dei responsi pitici di Erodoto. Nei testi degli oracoli

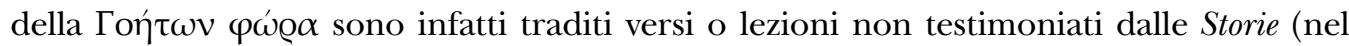
caso, ovvero, di PW29 [= PW216], PW53 e PW95), i quali sono talvolta confermati da altre fonti indipendenti da Enomao e a lui antecedenti. Rispetto ad Erodoto, i testi trascritti da

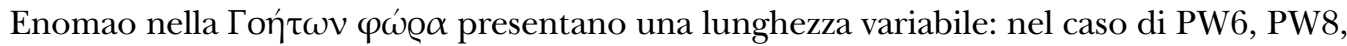
PW63, PW92, PW100, le versioni brevi degli oracoli, testualmente conformi ai brani di Erodoto, potrebbero essere il risultato di un personale sezionamento per mano del filosofo cinico, seppure non si possa escludere che esse fossero già decurtate nelle fonti consultate da Enomao; riguardo a PW94-95, cioè i due oracoli delfici agli Ateniesi, il fatto di trovare delle versioni accorciate ed anche discrepanti da un punto di vista formale e stilistico rispetto alle pregevoli versioni erodotee sembra più il riflesso di una fonte di mediocre qualità che non il frutto di una operazione di segmentazione dei testi di Erodoto.

È inoltre difficile che Enomao possa essere stato l'artefice degli oracoli non altrimenti attestati nella letteratura antica. L'analisi complessiva delle fonti conservate lascia intravedere l'esistenza di tradizioni letterarie più ampie rispetto alle Storie riguardo ai responsi ed alle afferenti narrazioni delle predizioni di Apollo Pitico. Sebbene Erodoto sia stato spesso utilizzato da vari autori successivi, varianti dei testi degli oracoli e molti altri responsi dovevano essere disponibili in epoca antica. Ciò è rilevabile nel caso delle tradizioni relative ai Cipselidi e a Creso: rispetto alle Storie, di esse si conservano delle versioni inedite di oracoli grazie ad Enomao e ad altri testimonia, nonché le notizie alternative di Nicola di Damasco, il quale dipende forse da Ellanico di Mitilene e da Androzione riguardo alla leggenda su Cipselo (PW7-8), ${ }^{71}$ e da Xanthos di Sardi a proposito del racconto di Creso sulla pira $(\mathrm{PW} 56) .{ }^{72}$ Una varietà di testi trattavano poi, ben più dettagliatamente di Erodoto, dell'ordinamento politico-legislativo di Sparta e della sua origine pitica, della quale Diodoro ed Enomao trasmettono alcuni responsi pronunciati a Licurgo prima della rivelazione della Rhetra da parte del dio.

Non si vede perché bisogna ritenere che Enomao abbia necessariamente inventato gli oracoli non altrimenti noti, come è stato postulato ancora a proposito del breve verso su Cipselo (PW9) riferito nella Гớ $َ \omega v \varphi \omega \omega \varrho \alpha$ come esempio dell'elogio dei tiranni e dei tirannicidi da parte di Apollo, ${ }^{73}$ e giudicato come un'invenzione e una versione satirica

71 Nic. Dam. 57, 2 (6) J, riguardo a PW7, mentre Nic. Dam. 57, 4 J, relativamente a PW8: cfr. Fontenrose (1978: p. 116).

72 Nic. Dam. 68, 13 J, riguardo a PW56. Secondo Fontenrose (1978: pp. 112-113), “[...] the pyre story with its antecedents was already current in Greek oral and written tradition in the fifth century, and both Herodotos and Xanthos incorporated it in their histories".

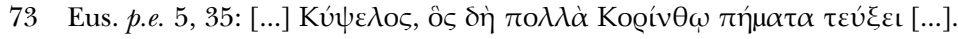


del secondo verso di PW8. ${ }^{74}$ La tracciabilità nella tradizione a lui precedente di alcuni degli oracoli delfici scarsamente attestati (e non menzionati da Erodoto) appare, anzi, confermare che Enomao sia stato testimone attendibile e che egli abbia a tutti gli effetti attinto a delle fonti reali.

Del resto, sono tante le risposte che ancora oggi ci sfuggono sul processo di formazione degli oracoli nel mondo antico, sia riguardo ai canali di trasmissione impiegati nel percorso dal santuario di Delfi (fulcro del centro dell'oracolo) al mondo esterno, sia in relazione alla genesi e allo sviluppo della tradizione orale e scritta sugli oracoli delfici, specie per l'età più antica.

Dalle fonti apprendiamo, inoltre, del prestigio e dell'influenza di cui godevano, spe-

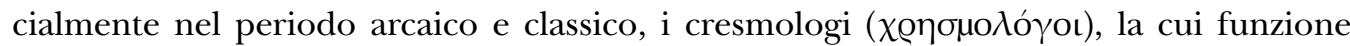
oscillava tra raccoglitori e proferitori di oracoli. ${ }^{75}$ Particolarmente richiesti soprattutto nelle forme di divinazione privata, essi venivano interrogati anche dalle poleis in occasione di guerre e di eventi di grande importanza: Tucidide ricorda come, agli inizi della guerra del Peloponneso, molti oracoli (logia) furono pronunciati dai cresmologi nelle città belligeranti e in quelle neutrali, e di come essi intonarono profezie di tutti i generi, a seconda delle disposizioni di spirito dei vari ascoltatori, al momento dell'invasione di Atene da parte dell'esercito spartano guidato da Archidamo, ${ }^{76}$ un sardonico attacco all'intera classe di indovini e di cresmologi è parimenti sferrato nelle commedie

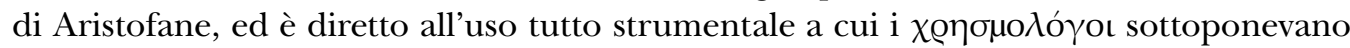
gli oracoli - reali ovvero costruiti ad arte per le singole occasioni - confluiti nelle loro collezioni. ${ }^{77}$ Di tali figure di indovini, ai quali specialmente Tucidide ed Aristofane non risparmiano una critica demolitrice e sarcastica, ci sono giunti alcuni nomi ${ }^{78}$ ed essi dovettero contribuire ad arricchire, attraverso le proprie raccolte di oracoli, la tradizione letteraria sull'oracolistica nel mondo greco. ${ }^{79}$

74 Tale è il parere di Fontenrose (1978: p. 288). Il verso è considerato parimenti spurio, ma con maggiore riserva, da Saarmann (1889: p. 33) e da Mras (1954: p. 288).

75 Sulle figure dei cresmologi e sulle forme di divinazione privata cfr. Parke \& Wormell (1956a: pp. XIIXXI); Lévy (1997: pp. 345-365); Dover (1991: pp. 303-311); Asheri (1993: pp. 63-76); Giuliani (2000: pp. 5-20); Dillery (2005: pp. 167-231); Johnston (2008).

76 Th. 2, 8, 2; 2, 21, 3.

77 Cfr. Fontenrose (1978: pp. 154-157); Shapiro (1990: pp. 335-345); Giuliani (2001: pp. 25 ss.); Lange (2006: pp. 267 ss.).

78 Oltre al già citato Bakis - noto anche ad Erodoto -, Pausania ricorda anche gli oracoli del cresmologo Lykos nell'ambito delle guerre messeniche: Paus. 4, 20, 4. Erodoto menziona, tra gli altri, i cresmologi Onomakritos di Atene e Amphilytos di Arcanania, i quali ebbero ambedue grande influenza sui Pisistratidi: Hdt. 1, 62, 4; 7, 6, 3-5. Aristofane stigmatizza l'attività del chresmologos Diopeithes, che fu autorevole ad Atene e a Sparta (cfr. Ar. Eq. 1084-1085; Id. V. 380; Id. Av. 988), e richiama, nella Pace, il profeta Stilbides ed il cresmologo Ierocle, le cui consultazioni occorrono in occasione del sacrificio di Trygaios ad Eirene nell'allora pacificata Atene (Ar. Pax 1026-1032; 1043-1126). Il chresmologos, cioè colui che proferiva o raccoglieva gli oracoli, era probabilmente figura diversa dal mantis, interprete di sogni e presagi, ispirato direttamente dalla divinità: cfr. Fontenrose (1978: p. 153).

79 Ad es., secondo Erodoto (Hdt. 7, 6, 3-5) il cresmologo ateniese Onomakritos fu diathetes degli oracoli di Musaios, e venne espulso da Atene per aver inserito in tale raccolta una profezia spuria, secondo la quale le isole lontane da Lemno sarebbero state inghiottite dal mare; Antichares di Eleone possedeva, invece, 
Oltre ai libri di responsi attribuiti a famosi cresmologi e indovini, in età antica vi erano molte altre collezioni di oracoli, non solo private ma anche pubbliche. Erodoto ci informa, ad esempio, della collezione di oracoli dei Pisistratidi custodita sull'Acropoli, la quale, dopo la caduta della tirannide intorno al 510, fu portata via da Cleomede a Sparta. ${ }^{80}$ Una collezione di oracoli delfici era posseduta anche dai re spartani e custodita da due ufficiali chiamati Pythioi ${ }^{81}$ Plutarco attesta che il testo dell'oracolo a Licurgo era custodito negli archivi più antichi di Sparta (anagraphai). ${ }^{82}$ È noto, peraltro, quanto ampia fu la diffusione di varie collezioni di oracoli sibillini, soprattutto nel mondo romano, molte delle quali erano composte da responsi della Sibilla di Delfi. ${ }^{83}$

Si delinea, dunque, una maglia articolata di canali di divulgazione di oracoli nel mondo antico, in funzione della quale appare necessario estirpare la convinzione che Erodoto - ritenuto ora rappresentante fedele della propaganda apologetica del clero di Delfi, ${ }^{84}$ ora forgiatore egli stesso degli oracoli delfici ${ }^{85}$ - debba considerarsi come modello archetipico per ogni tradizione successiva riguardante gli oracoli attestati nelle Storie, come più volte ritenuto dagli interpreti. In ambito letterario, oltre alle varie fonti che citavano a proprio piacimento i singoli responsi, la circolazione di una possibile "vulgata" degli oracoli delfici non esclude che autori successivi potessero rileggere, rielaborare o anche creare ex novo testi e versi attribuiti all'oracolo di Delfi.

Alla luce di tali osservazioni, è verosimile che per gli oracoli esaminati Enomao di Gadara non dipenda dalle Storie di Erodoto, le quali non vengono da lui mai menzionate. Negli estratti della Гó́ $\tau \omega \nu \varphi \omega ́ \mathrm{Q} \alpha$ nessuna fonte è in realtà indicata, nemmeno nel caso degli altri numerosi oracoli storici passati in rassegna, alcuni dei quali sono a noi noti mediante autori come Pausania, Ateneo, Eliano o lo Pseudo-Plutarco, altri invece del tutto sconosciuti. ${ }^{86}$ Le versioni degli oracoli di Delfi negli excerpta di Enomao differiscono testualmente dai brani di Erodoto, e probabilmente dovettero essere prelevate dal filosofo cinico da ben altri scritti che - benché affini per molti aspetti alla tradizione trasmessa da Diodoro Siculo - appaiono per noi indeterminabili, vista anche la ricchezza di fonti sulla mantica delfica disponibili nel mondo antico.

un libro di oracoli da lui chiamati Laiou chresmoi, i quali erano forse attribuiti all'omonimo re tebano, padre di Edipo (Hdt. 5, 43).

80 Hdt. 5, 90, 2.

81 Hdt. 6, 57, 2, 4.

82 Plu. mor. $1116 f$.

83 Oltre alle edizioni, con traduzione e bibliografia essenziale, di Collins (1983: pp. 317-472), Lightfoot (2007) e Monaca (2008), sulla letteratura sibillina di età greca e romana si veda Schwartz (1976: pp. 413420); Collins (1987: pp. 421-453); Caerols Pérez (1991); Cervelli (1993: pp. 895-1001); Sfameni Gasparro (1998: pp. 505-553; 2002); Monaca (2005).

84 Defradas (1954: p. 284).

85 Crahay (1956: p. IX).

86 Al riguardo un prospetto completo è fornito da des Places (1980: pp. 11-14). Anche quando sembra accennare alle sue fonti sugli oracoli, Enomao rimane sempre sul vago: nel passo di Eus. p.e. 5, 35, 2, il filosofo cinico afferma di aver conosciuto un (non altrimenti noto) oracolo pitico in prosa dedicato a Falaride, tiranno di Agrigento, il quale sarebbe stato lodato per l'umanità dimostrata nei confronti dei suoi cospiratori. 
Enomao di Gadara appare un testimone prezioso di formae vulgatae e di versioni orali degli oracoli di Delfi le quali, circolanti in età imperiale in aggiunta a fonti tradizionali come Erodoto, permettono di recuperare passaggi importanti nel processo di trasmissione degli oracoli pitici dall'età classica a quella tardoantica.

La critica razionalistica del filosofo cinico, condotta attraverso la raccolta composita e attenta dei testi degli oracoli, rivela che nel corso del II secolo non era venuta meno la fiducia nei confronti della religione oracolare ${ }^{87}$ la quale, grazie all'irruzione dell'irrazionale e del sincretismo nel coevo mondo ellenistico-romano, continuò a mantenere vitalità ancora per qualche tempo, a dispetto della progressiva perdita di prestigio e del declino del centro di culto di Delfi. ${ }^{88}$

\begin{tabular}{|c|c|}
\hline \multicolumn{2}{|c|}{ PW6 = Q59 } \\
\hline Hdt. $5,92 \beta$ & Oenomaus Gadarenus: Eus. p.e. 5, 29, 2, 2-3 \\
\hline 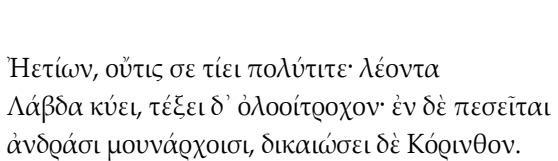 & 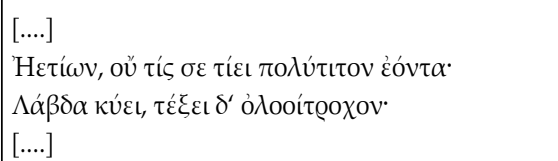 \\
\hline
\end{tabular}

\begin{tabular}{|c|c|}
\hline \multicolumn{2}{|c|}{ PW8 = Q61 } \\
\hline Hdt. $5,92 \varepsilon$ & Oenomaus Gadarenus: Eus. p.e. 5, 35, 1, 1-2 \\
\hline 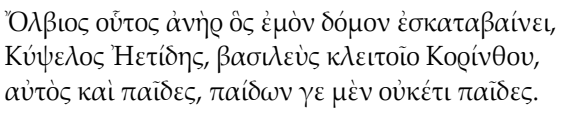 & 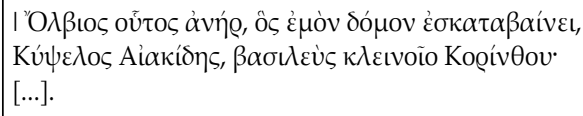 \\
\hline
\end{tabular}

87 La posizione scettica e caustica di Enomao di Gadara nei confronti dell'oracolo delfico si pone d'altra parte in contrasto con l'operazione culturale opposta e simmetrica intrapresa dal contemporaneo Plutarco, che fu membro del collegio sacerdotale del santuario di Delfi e rappresentante della teologia delfica: cfr. in merito Lamberton (2001: pp. 52-59); Casanova (2012: pp. 151-157).

88 Sulla “fine” dell'oracolo delfico cfr. Guida (2000: pp. 389-413). 
Versioni e formae vulgatae degli oracoli di Delfi: alcune note a proposito di Erodoto ...

\begin{tabular}{|c|c|}
\hline \multicolumn{2}{|c|}{ PW29 / PW216 = Q7 } \\
\hline Hdt. $1,65,3$ & Oenomaus Gadarenus: Eus. p.e. 5, 27, 8, 5-8 \\
\hline \multirow[t]{3}{*}{ 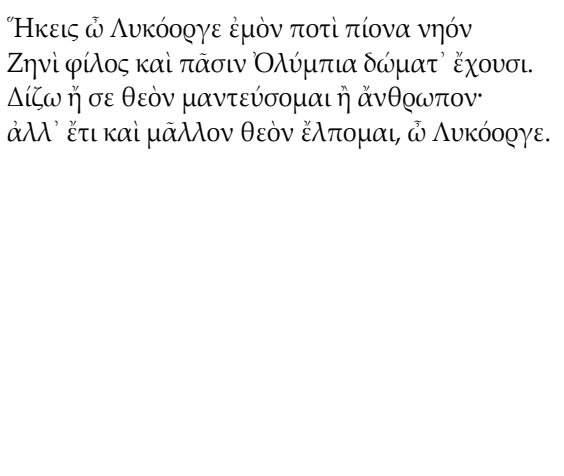 } & 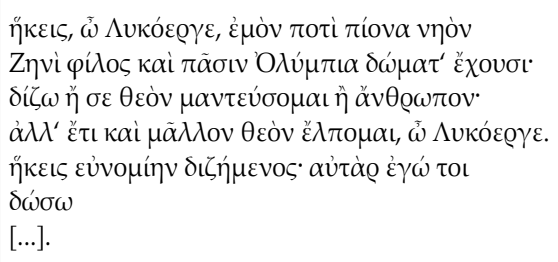 \\
\hline & Oenomaus Gadarenus: Eus. p.e. 5, 28, 2, 7-8 \\
\hline & 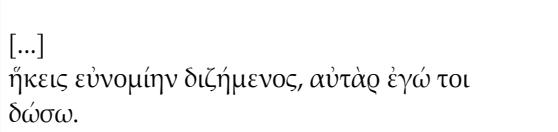 \\
\hline
\end{tabular}

\begin{tabular}{|c|c|}
\hline \multicolumn{2}{|c|}{ PW 52 = Q99 } \\
\hline Hdt. $1,47,3$ & Oenomaus Gadarenus: Eus. p.e. 5, 21, 1 \\
\hline \multirow[t]{3}{*}{ 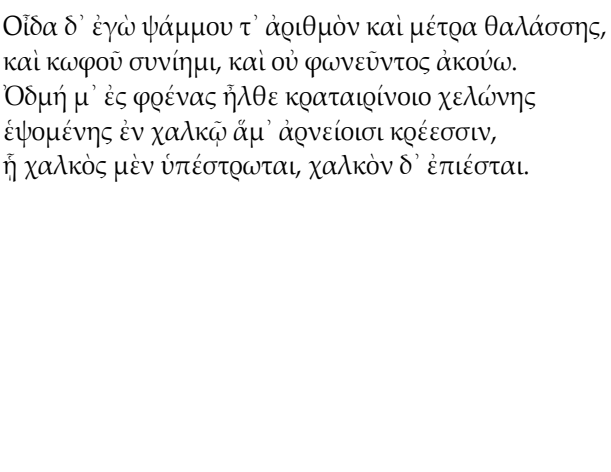 } & 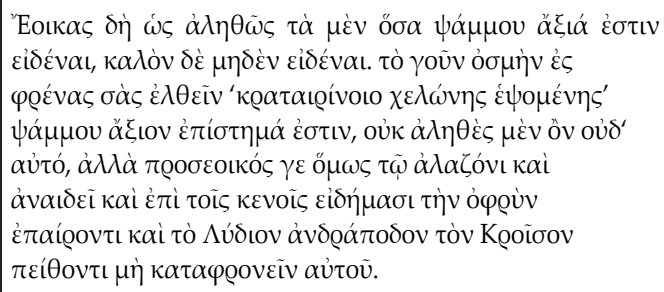 \\
\hline & Oenomaus Gadarenus: Eus. p.e. 5, 34, 2, 1-2 \\
\hline & 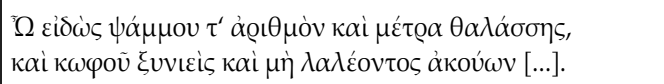 \\
\hline
\end{tabular}




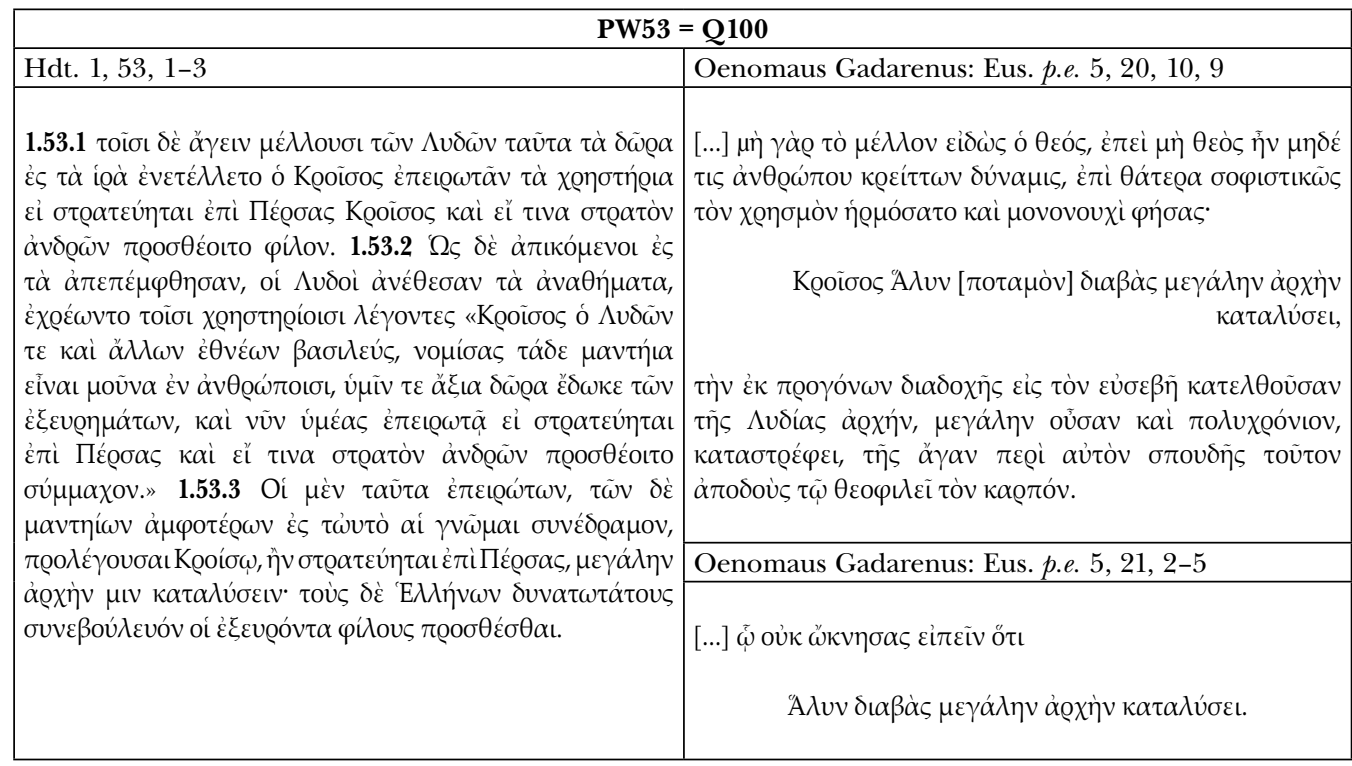

\begin{tabular}{|c|c|}
\hline \multicolumn{2}{|c|}{ PW54 = Q101 } \\
\hline Hdt. 1,55 & Oenomaus Gadarenus: Eus. p.e. 5, 21, 4, 1-3 \\
\hline \multirow[t]{3}{*}{ 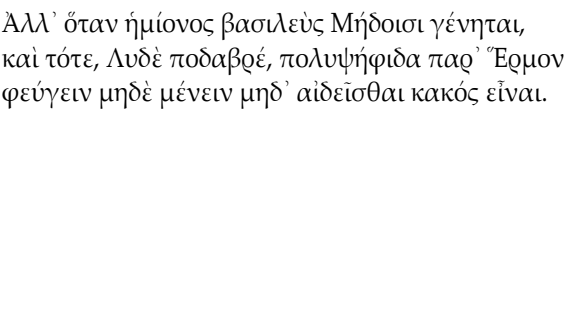 } & 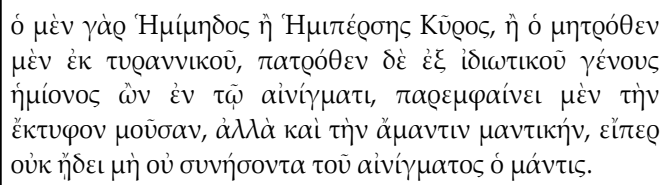 \\
\hline & Oenomaus Gadarenus: Eus. p.e. 5, 24, 8, 7 \\
\hline & 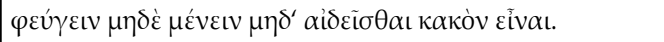 \\
\hline
\end{tabular}

\begin{tabular}{|c|c|}
\hline \multicolumn{2}{|c|}{ PW63 = Q112 } \\
\hline Hdt. 1, 174 & Oenomaus Gadarenus: Eus. p.e. 5, 26, 2, 2-3 \\
\hline 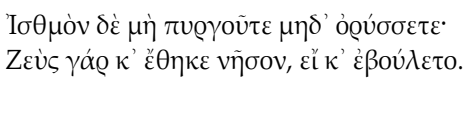 & 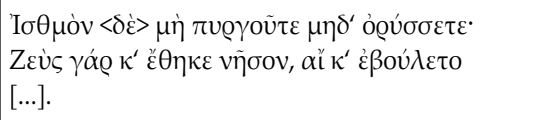 \\
\hline
\end{tabular}


Versioni e formae vulgatae degli oracoli di Delfi: alcune note a proposito di Erodoto ...

\begin{tabular}{|c|c|}
\hline \multicolumn{2}{|c|}{ PW92 = Q144 } \\
\hline Hdt. $7,148,3$ & Oenomaus Gadarenus: Eus. p.e. 6, 7, 4, 3-4 \\
\hline 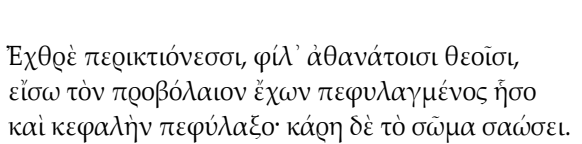 & 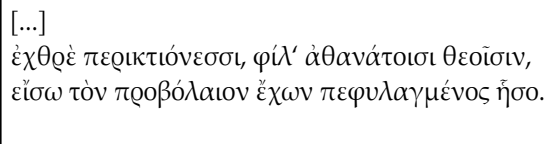 \\
\hline
\end{tabular}

\begin{tabular}{|c|c|}
\hline \multicolumn{2}{|c|}{ PW94 = Q146 } \\
\hline Hdt. $7,140,2-3$ & Oenomaus Gadarenus: Eus. p.e. 5, 24, 1, 4-11 \\
\hline 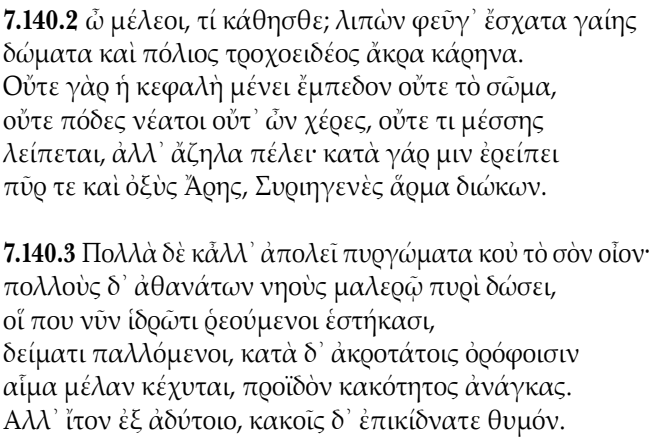 & 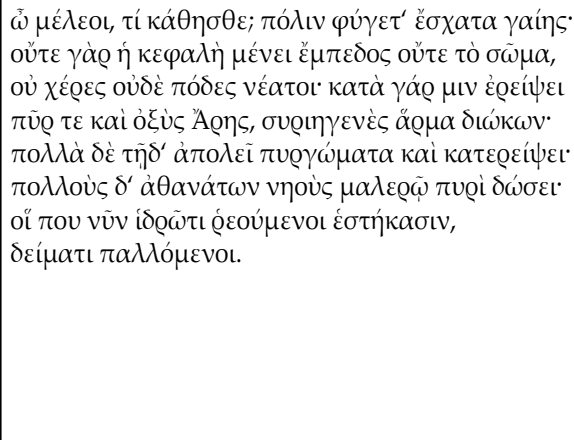 \\
\hline
\end{tabular}

\begin{tabular}{|c|c|}
\hline \multicolumn{2}{|c|}{ PW95 = Q147 } \\
\hline Hdt. 7, 141, 3-4 & Oenomaus Gadarenus: Eus. p.e. 5, 24, 2, 5-14 \\
\hline 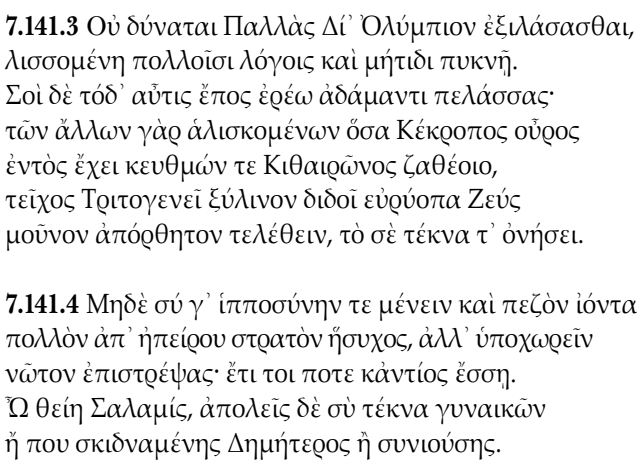 & 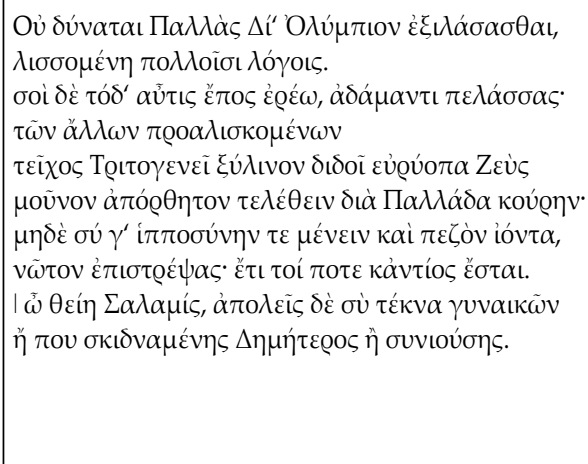 \\
\hline
\end{tabular}




\begin{tabular}{|c|c|}
\hline \multicolumn{2}{|c|}{ PW100 = Q152 } \\
\hline Hdt. $7,220,4$ & Oenomaus Gadarenus: Eus. p.e. 5, 25, 2, 3-6 \\
\hline 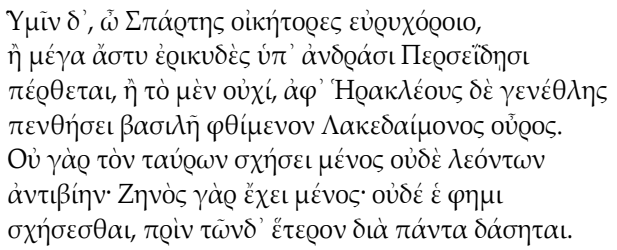 & 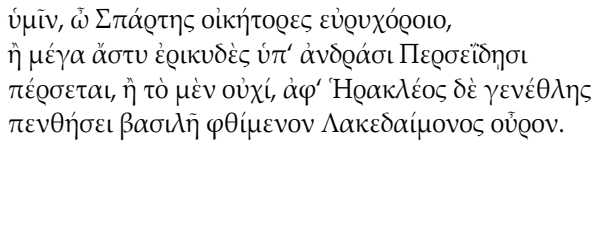 \\
\hline
\end{tabular}

\section{Bibliography}

\section{Primary sources}

Collins, J. J. (1983). Sibylline Oracles. Second Century B.C. - Seventh Century A.D. In J. H. Charlesworth (Ed.), The Old Testament Pseudepigrapha, Vol. I.: Apocalyptic Literature and Testaments (pp. 317-472). New York: Doubleday.

Colonna, A., \& Bevilacqua, F. (Eds.). (1996). Le Storie di Erodoto (Vol. I-II). Torino: Utet.

des Places, É. (Ed.). (1980). Eusèbe de Césarée. La Préparation Évangélique (Livres V.18-36-VI; Sources chrétiennes, 266). Paris: Éditions du Cerf.

Gentili, B., \& Prato, C. (1979). Poetae Elegiaci. Testimonia et Fragmenta (Pars prior). Leipzig: Bibliotheca Teubneriana.

Gressmann, H. (Ed.). (1904). Eusebius' Werke, 3.2: Die Theophanie. Die griechischen Bruchstücke und Übersetzung der syrischen Überlieferungen (Die griechischen christlichen Schriftsteller der ersten drei Jahrhunderte, 11.2). Leipzig: Hinrichs.

Hammerstaedt, J. (1988). Die Orakelkritik des Kynikers Oenomaus. Frankfurt am Main: Athenäum.

Legrand, Ph.-E. (Ed.). (1951). Herodotus: Polymnie. Paris: Les Belles Lettres.

Lightfoot, J. L. (2007). The Sibylline Oracles with Introduction, Translation, and Commentary on the First and Second Books. Oxford: Oxford University Press.

Meriani, A., \& Giannattasio Andria, R. (Eds.). (1998). Vite di Plutarco (Vol. sesto). Torino: Utet.

Migliore, F. (2012). Eusebio di Cesarea. Preparazione Evangelica (Vol. I-III). Roma: Città Nuova.

Monaca, M. (Ed.). (2008). Oracoli sibillini. Roma: Città Nuova.

Mras, K. (Ed.). (1954-1956 [1982²]). Eusebius' Werke, 8: Die Praeparatio Evangelica (Die griechischen christlichen Schriftsteller der ersten drei Jahrhunderte, 43.1-2). Berlin: Akademie Verlag.

Mullach, F. G. A. (Ed.). (1867). Fragmenta Philosophorum Graecorum (= FPhG) (Vol. II). Paris: Firmin-Didot.

Nenci, G. (Ed.). (1994). Erodoto. Le Storie. Libro V. La rivolta della Ionia (Fondazione Lorenzo Valla). Milano: Arnoldo Mondadori Editore.

Oldfather, C. H. (Ed.). (1952). Diodorus of Sicily (Vol. III., Books IV.59-VIII). Cambridge (Mass.): Harvard University Press.

Parke, H. W., \& Wormell, D. E. W. (1956). The Delphic Oracle, I: The History. Oxford: Blackwell. 
Parke, H. W., \& Wormell, D. E. W. (1956a). The Delphic Oracle, II: The Oracular Responses. Oxford: Blackwell.

Pini, G. (Ed.). (1985). Clemente Alessandrino. Stromati, note di vera filosofia. Milano: Paoline.

Pontani, F. M. (Ed.). (1981). Antologia Palatina (Vol. quarto, Libri XII-XVI). Torino: Giulio Einaudi Editore.

Zink, O. (Ed.). (1979). Eusèbe de Césarée. La Préparation Évangélique (Livres IV-V, 1-17; Sources chrétiennes, 262). Paris: Éditions du Cerf.

\section{Secondary sources}

Addey, C. (2014). Divination and Theurgy in Neoplatonism. Oracles of the Gods. Farnham - Burlington (VT): Ashgate.

Asheri, D. (1993). Erodoto e Bacide. Considerazioni sulla fede di Erodoto negli oracoli (Hdt. VIII 77). In M. Sordi (Ed.), La profezia nel mondo antico (pp. 63-76). Milano: CISA.

Attridge, A. H. (1978). Philosophical Critique of Religion under the Early Empire. In H. Temporini (Ed.), Aufstieg und Niedergang der römischen Welt (Vol. II, 16.1; pp. 45-78). Berlin - New York: De Gruyter.

Barker, E. (2006). Paging the oracle: interpretation, identity and performance in Herodotus' History. Greece E Rome, 53(1), 1-28.

Bettalli, M. (Ed.). (2001). Introduzione alla storiografia greca. Roma: Carocci.

Bonnechere, P. (2006). La divination apollinienne sous l'Empire. Un dossier opportunément rouvert. Kernos, 19, 447-522.

Brancacci, A. (2000). Libertà e fato in Enomao di Gadara. In A. Brancacci (Ed.), La filosofia in età imperiale. Le scuole e le tradizioni filosofiche ("Elenchos", XXXI; pp. 37-67). Napoli: Bibliopolis.

Brancacci, A. (2001). La polemica antifatalistica di Enomao di Gadara. In A. Brancacci (Ed.), Antichi e moderni nella filosofia di età imperiale: atti del II colloquio internazionale. Roma, 21-23 settembre 2000 (pp. 71-110). Napoli: Bibliopolis.

Busine, A. (2005). Paroles d'Apollon: Practiques et traditions oraculaires dans l'Antiquité tardive (II -VI siècles). Leiden - Boston: Brill.

Busine, A. (2014). Le problème de l'attribution de textes oraculaires au sanctuaire de Claros. In J.C. Moretti (Ed.), Le sanctuaire de Claros et son oracle. Actes du colloque international de Lyon, 13-14 Janvier 2012. Lyon: Maison de l'Orient et de la Méditerranée.

Caerols Pérez, J. J. (1991). Los Libros Sibillinos en la Historiografia Latina. Diss. Madrid.

Casanova, A. (2012). Plutarch as Apollo's Priest at Delphi. In L. Roig Lanzillotta, \& I. Muñoz Gallarte (Eds.), Plutarch in the Religious and Philosophical Discourse of Late Antiquity (pp. 151-157). Leiden - Boston: Brill.

Cervelli, I. (1993). Questioni sibilline. Studi Storici, 4, 895-1001.

Collins, J. J. (1987). The Development of the Sibylline Tradition. In H. Temporini (Ed.), Aufstieg und Niedergang der römischen Welt (Vol. II, 20.1; pp. 421-453). Berlin: De Gruyter.

Crahay, R. (1956). La littérature oraculaire chez Hérodote (pp. 182-207). Paris: Belles Lettres.

Defradas, J. (1954). Les thèmes de la propagande delphique. Paris: Klincksieck.

Dillery, J. (2005). Chresmologues and Manteis: Independent Diviners and the Problem of Autho- 
rity. In S. I. Johnston, \& P. T. Struck (Eds.), Mantikê: Studies in Ancient Divination (pp. 167-231). Leiden: Brill.

Dover, K. (1991). Gli oracoli in Tucidide. In Studi di filologia classica in onore di Giusto Monaco (Vol. I; pp. 303-311). Palermo: Università di Palermo.

Dudley, D. R. (1937). A History of Cynism. From Diogenes to the 6th Century A.D. London: Methuen \& Co.

Fehling, D. (1989). Herodotus and his 'sources'. Citation, invention and narrative art (Ed. and transl. J. G. Howie). Leeds: Leeds Latin Seminar.

Fontenrose, J. (1978). The Delphic Oracle. Its Responses and Operations with a Catalogue of Responses. Berkeley - Los Angeles - London: Univ. of California Press.

Fowler, R. L. (1996). Herodotos and his contemporaries. Journal of Hellenic Studies, 116, 62-87.

Gagné, R. (2013). Poétiques de la chrèsmodie. L'oracle de Glaukos (Hérodote, VI, 86). Kernos, 26, 95-109.

Giuliani, A. (2000). Erodoto, Tucidide e gli indovinelli degli indovini. Considerazioni sull'ambiguità del linguaggio oracolare. Aevum, 74, 5-20.

Giuliani, A. (2001). La città e l'oracolo. I rapporti tra Atene e Delfi in età arcaica e classica. (Pubblicazioni dell'Università Cattolica del Sacro Cuore). Milano: Vita e Pensiero.

Guida, A. (2000). L'ultimo oracolo di Delfi per Giuliano. In Aa.Vv., Giuliano imperatore: le sue idee, i suoi amici, i suoi avversari. Atti del Convegno Internazionale di Studi, Lecce 10-12 dicembre 1998 (pp. 389-413). Lecce: Brossura.

Hammerstaedt, J. (1993). Le cynisme littéraire à l'époque imperial. In M.-O. Goulet-Cazé, \& R. Goulet (Eds.), Le cynisme ancien et ses prolongements. Actes du Colloque International du CNRS, Paris, 22-25 juillet 1991 (pp. 399-418). Paris: P.U.F.

Hammond, N. G. L. (1959). A History of Greece to 322 B.C. Oxford: Oxford University Press.

Hartog, F. (1980). Le miroir d'Hérodote. Essai sur la représentation de l'autre. Paris: Gallimard.

Hignett, C. (1963). Xerxes' Invasion of Greece. Oxford: Oxford Clarendon Press.

Inowlocki, S. (2006). Eusebius and the Jewish Authors. His Citation Technique in an Apologetic Context. Leiden - Boston: Brill.

Johnston, S. I. (2008). Ancient Greek Divination. Oxford: Blackwell.

Kindt, J. (2006). Delphic Oracle Stories and the Beginning of Historiography: Herodotus' Croesus Logos. Classical Philology, 101(1), 34-51.

Lamberton, R. (2001). Plutarch. New Haven, Conn.: Yale University Press.

Lange, A. (2006). Literary Prophecy and Oracle Collection: A Comparison between Judah and Greece in Persian Times. In M. Floyd, \& R. D. Haak (Eds.), Prophets, Prophecy, and Prophetic Texts in Second Temple Judaism (pp. 248-275). New York - London: T\&T Clark International.

Lévy, E. (1997). Devins et oracles chez Hérodote. In J.-G. Heintz (Ed.), Oracles et prophéties dans l'Antiquité, Actes du Colloque de Strasbourg 15-17 juin 1995 (pp. 345-365). Paris: de Boccard.

Luraghi, N. (1994). Erodoto tra storia e fantasia: la parola alla difesa. Quaderni di storia, 40, 181190.

Maurizio, L. (1993). Delphic Narratives: Recontextualizing the Pythia and her Prophecies. Diss. Stanford University.

Maurizio, L. (1997). Delphic Oracles as Oral Performances: Authenticity and Historical Evidence. Classical Antiquity, 16, 308-334. 
Meyer, E. (1892). Forschungen zur alten Geschichte (Vol. I). Halle: Niemeyer.

Monaca, M. (2005). La Sibilla a Roma: i libri sibillini fra religione e politica. Cosenza: Giordano.

Nagy, G. (1996). Poetry as Performance: Homer and Beyond. Cambridge: Cambridge University Press.

Panitz, H. (1935). Mythos und Orakel bei Herodot. Greifswald: Dallmeyer.

Rainart, G. (2014). La langue de l'Apollon de Delphes: analyse linquistique, poétique et systématique des recueils d'oracles. Université Nice Sophia Antipolis.

Saarmann, Th. (1887). De Oenomao Gadareno. Diss. Tubingae.

Saarmann, Th. (1889). Adnotationes ad Oenomai Cynici fragmenta. Dortmund: Diss. Tubingae.

Sardella, T. (1988). Apollo, Istaspe e la Sibilla: la cristianizzazione degli oracoli pagani da Giustino a Clemente. In Hestiasis. Studi di tarda antichità offerti a Salvatore Calderone (pp. 295-329). Messina: Sicania.

Schwartz, J. (1976). L'historiographie impériale des Oracula Sibyllina. Dialogues d'histoire ancienne, 2, 413-420.

Sfameni Gasparro, G. (1998). La Sibilla voce del dio per pagani, ebrei e cristiani: un modulo profetico al crocevia delle fedi. In I. Chirassi Colombo, \& T. Seppilli (Eds.), Sibille e linguaggi oracolari. Mito, storia, tradizione. Atti del Convegno, Macerata - Norcia settembre 1994 (pp. 505-553). Pisa - Roma: Istituti Editoriali e Poligrafici Internazionali.

Sfameni Gasparro, G. (2002). Oracoli Profeti Sibille. Rivelazione e salvezza nel mondo antico. Roma: Libreria Ateneo Salesiano.

Shapiro, H. A. (1990). Oracle-Mongers in Peisistratid Athens. Kernos, 3, 335-345.

Vallette, P. (1908). De Oenomao Cynico. Diss. Paris.

Zamagni, C. (2010). Alexandre Polyhistor et Artapan: une mise en perspective à partir des extraits d'Eusèbe de Césarée. In Ph. Borgeaud, \& Th. Römer (Eds.), Interprétations de Moïse. Égypte, Judée, Grèce et Rome (pp. 57-82). Leiden - Boston: Brill.

Cristian Mondello, Ph.D. / cristianmondello@libero.it

Dipartimento di Civiltà Antiche e Moderne

Università di Messina

Viale Annunziata, Polo universitario, 98168 Messina, Italia 